\title{
A divided differences based medium to analyze smoothness of the binary bivariate refinement schemes
}

\author{
Rabia Hameed ${ }^{1}$, Ghulam Mustafa² ${ }^{2}$, Dumitru Baleanu ${ }^{3,4}$ and Yu-Ming Chu ${ }^{5 *}$
}

\section{"Correspondence:}

chuyuming2005@126.com

${ }^{5}$ Department of Mathematics,

Huzhou University, 313000 Huzhou,

P.R. China

Full list of author information is

available at the end of the article

\section{Springer}

\begin{abstract}
In this article, we present the continuity analysis of the 3D models produced by the tensor product scheme of $(m+1)$-point binary refinement scheme. We use differences and divided differences of the bivariate refinement scheme to analyze its smoothness. The $C^{0}, C^{1}$ and $C^{2}$ continuity of the general bivariate scheme is analyzed in our approach. This gives us some simple conditions in the form of arithmetic expressions and inequalities. These conditions require the mask and the complexity of the given refinement scheme to analyze its smoothness. Moreover, we perform several experiments by using these conditions on established schemes to verify the correctness of our approach. These experiments show that our results are easy to implement and are applicable for both interpolatory and approximating types of the schemes.
\end{abstract}

MSC: 65D17;65D15; 65D10;60E05; 62 H10

Keywords: Refinement scheme; Divided difference; Smoothness; Continuity; Subdivision scheme; Inequalities; Bernstein polynomial

\section{Introduction}

Refinement schemes are used for curve and surface modeling. These schemes require the initial data or initial polygons/mesh as input, smooth the data several times by its recursive refinement rules and give smooth limiting shapes as output. The goodness of the refinement scheme can be measured by the order of its smoothness. For instance, a $C^{2}$ continuous refinement scheme is superior over a $C^{1}$-continuous scheme. In the literature, the order of smoothness of the refinement schemes has been analyzed by various methods.

In the literature, inequalities have been used to present many important results. For instant, Agarwal et al. [1] presented a strategy to prove some new Pólya-Szegö type integral inequalities. They used these inequalities to formulate some fractional integral inequalities of Chebyshev type. Zhang et al. [2] gave the sufficient conditions for the existence and uniqueness of solutions for fractional differential systems by applying the generalized Gronwall inequality. Saoudi et al. [3] used inequalities to present the existence of solutions to the boundary value problem for the nonlinear fractional differential equations. Sitho et al. [4] presented some new non-instantaneous impulsive inequalities us-

(c) The Author(s) 2021. This article is licensed under a Creative Commons Attribution 4.0 International License, which permits use sharing, adaptation, distribution and reproduction in any medium or format, as long as you give appropriate credit to the original author(s) and the source, provide a link to the Creative Commons licence, and indicate if changes were made. The images or other third party material in this article are included in the article's Creative Commons licence, unless indicated otherwise in a credit line to the material. If material is not included in the article's Creative Commons licence and your intended use is not permitted by statutory regulation or exceeds the permitted use, you will need to obtain permission directly from the copyright holder. To view a copy of this licence, visit http://creativecommons.org/licenses/by/4.0/. 
ing the conformable fractional calculus. Jain et al. [5] discussed various estimates by the Hermite-Hadamard inequality for functions whose absolute values of the second derivatives to positive real powers are log-convex. Tomar et al. [6] established some generalized Hermite-Hadamard inequalities for generalized convex functions. They also presented certain interesting inequalities involving generalized arithmetic and logarithmic means.

The representation of the subdivision mask in form of the Laurent polynomials was introduced in [7]. The well-known Laurent polynomial method was firstly introduced by Dyn [7] to analyze the convergence and smoothness of the curves and surfaces generated by linear uniform refinement schemes. This method requires the subdivision mask and factorization of the Laurent polynomial of the given refinement scheme. The Laurent polynomial method with several modifications was used to analyze the smoothness of the non-uniform linear binary refinement scheme by Levin [8]. Dyn and Wallner [9] analyzed the smoothness of the subdivision curves produced by linear and its corresponding nonlinear refinement schemes. He used conditions on the smoothness of the linear scheme to analyze the smoothness of its corresponding nonlinear refinement scheme.

$\mathrm{Qu}$ [10-12] introduced the "divided differences" and "cross difference of directional divided differences" techniques to analyze the schemes. The analysis of the convergence of curve refinement scheme based on the contractiveness of its corresponding difference scheme was introduced by Dyn et al. [13]. They also give the smoothness analysis of the univariate refinement schemes based on the "divided difference". The smoothness analysis of the parametric refinement schemes was presented by [13-15]. Dyn et al. [15] presented the analysis for the regular portion of the butterfly scheme. Gregory [16] showed that, for $\omega \in\left(0, \frac{1}{12}\right)$, the butterfly scheme produces the $C^{1}$-smooth surfaces on regular grids.

Till then, the Laurent polynomial method was not mature enough to analyze the nonstationary and non-uniform schemes. Dyn and Levin $[17,18]$ presented the updated method based on Laurent polynomial for the analysis of non-stationary schemes. They have used the "divided difference" technique [19] for the analysis of non-uniform schemes. Guglielmi et al. [20] analyzed a 2-point Hermite refinement scheme in 2011. They have used the joint spectral radius of the matrices.

Nowadays, the Laurent polynomial technique is commonly used, but this technique has some limitations. In this technique, first a sequence of coefficients used in the refinement rules of the schemes is converted into the polynomial. Secondly, the polynomial is factorized. In this technique, multiplication, division, factorization of the polynomials are involved. Furthermore, the computation of inequalities and the comparison of the terms are also involved. In our technique, the comparison of four algebraic expressions is involved to compute the order of continuity. Furthermore, these algebraic expressions are made up of the constants used in the refinement rules of the schemes. Therefore our technique is easy to use. Our technique can be used to analyze the $(m+1)^{2}$-point Generalized Tensor Product Binary Refinement Scheme (GTPBRS). It is based on the study of differences and Generalized Bivariate Divided Difference Refinement Schemes (GBDDRS). It has been studied in a general setting up to $C^{2}$-continuity. The extension to higher order continuities can be done similarly.

The rest of this paper is structured as follows: In Sect. 2, the generalized expressions for tenor product binary and its corresponding GBDDRS are given. The necessary conditions for their convergence are also discussed in this section. In Sect. 3, the deviations between successive levels of polygons are derived. In Sect. 4, the inequalities to estimate the conti- 
nuity analysis of the binary bivariate refinement scheme are presented. Implementation of the proposed method is presented in Sect. 5. A summary and a conclusion of this research are presented in Sect. 6 .

\section{Tensor product binary and its GBDDRSs}

We start this section by introducing the GTPBRS. We fix $m$ to be 3 and derive the first and second order GBDDRSs of the 16-point tensor product refinement scheme. Moreover, we generalize these GBDDRSs for $(m+1)^{2}$ points. Furthermore, we calculate the tensor product of the first derivatives of Bernstein polynomials. We also compute the tensor product of second derivatives of Bernstein polynomials. In the last part of the section, we prove that the tensor product of first derivatives of Bernstein polynomials converges to the first order GBDDRS. And the tensor product of second derivatives of Bernstein polynomials converges to the second order GBDDRS.

\subsection{The GTPBRS}

Let $\varphi^{\psi}$ be the mesh obtained by joining the points $\left\{\varphi_{\sigma_{1}, \sigma_{2}}^{\psi} \in \mathbb{R}^{N}: \sigma_{1}, \sigma_{2} \in \mathbb{Z}\right\}$ where $\psi \in \mathbb{N}$ and $N$ is any integer whose value is restricted to be greater than 1 . Then, for an arbitrary $m$, the GTPBRS which is used to refine the given data is given below

$$
\left\{\begin{array}{l}
\varphi_{2 \sigma_{1}, 2 \sigma_{2}}^{\psi+1}=\sum_{r=0}^{m} \sum_{s=0}^{m} \rho_{r} \rho_{s} \varphi_{\sigma_{1}+r, \sigma_{2}+s}^{\psi} \\
\varphi_{2 \sigma_{1}, 2 \sigma_{2}+1}^{\psi+1}=\sum_{r=0}^{m} \sum_{s=0}^{m} \rho_{r} \chi_{s} \varphi_{\sigma_{1}+r, \sigma_{2}+s}^{\psi} \\
\varphi_{2 \sigma_{1}+1,2 \sigma_{2}}^{\psi+1}=\sum_{r=0}^{m} \sum_{s=0}^{m} \chi_{r} \rho_{s} \varphi_{\sigma_{1}+r, \sigma_{2}+s}^{\psi} \\
\varphi_{2 \sigma_{1}+1,2 \sigma_{2}+1}^{\psi+1}=\sum_{r=0}^{m} \sum_{s=0}^{m} \chi_{r} \chi_{s} \varphi_{\sigma_{1}+r, \sigma_{2}+s}^{\psi}
\end{array}\right.
$$

where

$$
\left\{\begin{array}{l}
\sum_{r=0}^{m} \rho_{r}=\sum_{r=0}^{m} \chi_{r}=1, \\
\sum_{r=0}^{m} \sum_{s=0}^{m} \rho_{r} \rho_{s}=\sum_{r=0}^{m} \sum_{s=0}^{m} \rho_{r} \chi_{s}=1, \\
\sum_{r=0}^{m} \sum_{s=0}^{m} \chi_{r} \rho_{s}=\sum_{r=0}^{m} \sum_{s=0}^{m} \chi_{r} \chi_{s}=1,
\end{array}\right.
$$

and $m \in \mathbb{N} \backslash\{0\}$.

The GTPBRS defined in (1) uses three types of points to refine each quadrilateral face. Thus in (1) $\varphi_{2 \sigma_{1}, 2 \sigma_{2}}^{\psi+1}$ is the vertex point, $\varphi_{2 \sigma_{1}+1,2 \sigma_{2}}^{\psi+1} \& \varphi_{2 \sigma_{1}, 2 \sigma_{2}+1}^{\psi+1}$ are the edge points and $\varphi_{2 \sigma_{1}+1,2 \sigma_{2}+1}^{\psi+1}$ is the face point. The bivariate refinement scheme (1) refines each quadrilateral face by inserting nine new points at each refinement step. Four of these are edge points, four are vertex points and one is the face point as discussed in Fig. 1.

If $\left\{\varphi_{\sigma_{1}, \sigma_{2}}^{0}: \sigma_{1}, \sigma_{2} \in \mathbb{Z}\right\}$ defines the initial control points in $\mathbb{R}^{N}$, then in the limit $k \rightarrow \infty$, the bivariate refinement scheme (1) gives an infinite number of points in $\mathbb{R}^{N}$. If $\varphi^{\psi}=$ $\left\{\left(\frac{\sigma_{1}}{2^{\psi}}, \frac{\sigma_{2}}{2^{\psi}}, \varphi_{\sigma_{1}, \sigma_{2}}^{\psi}\right): \sigma_{1}, \sigma_{2} \in \mathbb{Z}, N>1, \psi \in \mathbb{N}\right\}$ is the mesh at the $\psi$ th refinement step, then by using GTPBRS (1) one time we get the mesh $\varphi^{\psi+1}=\left\{\left(\frac{\sigma_{1}}{2^{\psi+1}}, \frac{\sigma_{2}}{2^{\psi+1}}, \varphi_{\sigma_{1}, \sigma_{2}}^{\psi+1}\right): \sigma_{1}, \sigma_{2} \in \mathbb{Z}, N>\right.$ $1, \psi \in \mathbb{N}\}$ at the $(\psi+1)$ th refinement step. For instance, $\varphi_{2 \sigma_{1}, 2 \sigma_{2}}^{\psi+1}, \varphi_{2 \sigma_{1}+2,2 \sigma_{2}}^{\psi+1}, \varphi_{2 \sigma_{1}, 2 \sigma_{2}+2}^{\psi+1}$ and $\varphi_{2 \sigma_{1}+2,2 \sigma_{2}+2}^{\psi+1}$ replaces the values $\varphi_{\sigma_{1}, \sigma_{2}}^{\psi}, \varphi_{\sigma_{1}+1, \sigma_{2}}^{\psi}, \varphi_{\sigma_{1}, \sigma_{2}+1}^{\psi}$ and $\varphi_{\sigma_{1}+1, \sigma_{2}+1}^{\psi}$ at the mesh points $\left(\frac{\sigma_{1}}{2^{\psi}}, \frac{\sigma_{2}}{2^{\psi}}\right),\left(\frac{\sigma_{1}+1}{2^{\psi}}, \frac{\sigma_{2}}{2^{\psi}}\right),\left(\frac{\sigma_{1}}{2^{\psi}}, \frac{\sigma_{2}+1}{2^{\psi}}\right)$ and $\left(\frac{\sigma_{1}+1}{2^{\psi}}, \frac{\sigma_{2}+1}{2^{\psi}}\right)$, respectively. The control points $\varphi_{2 \sigma_{1}+1,2 \sigma_{2}}^{\psi+1}$, $\varphi_{2 \sigma_{1}, 2 \sigma_{2}+1}^{\psi+1}, \varphi_{2 \sigma_{1}+1,2 \sigma_{2}+1}^{\psi+1}, \varphi_{2 \sigma_{1}+2,2 \sigma_{2}+1}^{\psi+1}$ and $\varphi_{2 \sigma_{1}+1,2 \sigma_{2}+2}^{\psi+1}$ are inserted at the new mesh points $\left(\frac{\sigma_{1}+1}{2^{\psi+1}}, \frac{\sigma_{2}}{2^{\psi+1}}\right),\left(\frac{\sigma_{1}}{2^{\psi+1}}, \frac{\sigma_{2}+1}{2^{\psi+1}}\right),\left(\frac{\sigma_{1}+1}{2^{\psi+1}}, \frac{\sigma_{2}+1}{2^{\psi+1}}\right),\left(\frac{\sigma_{1}+2}{2^{\psi+1}}, \frac{\sigma_{2}+1}{2^{\psi+1}}\right)$ and $\left(\frac{\sigma_{1}+1}{2^{\psi+1}}, \frac{\sigma_{2}+2}{2^{\psi+1}}\right)$, respectively. 


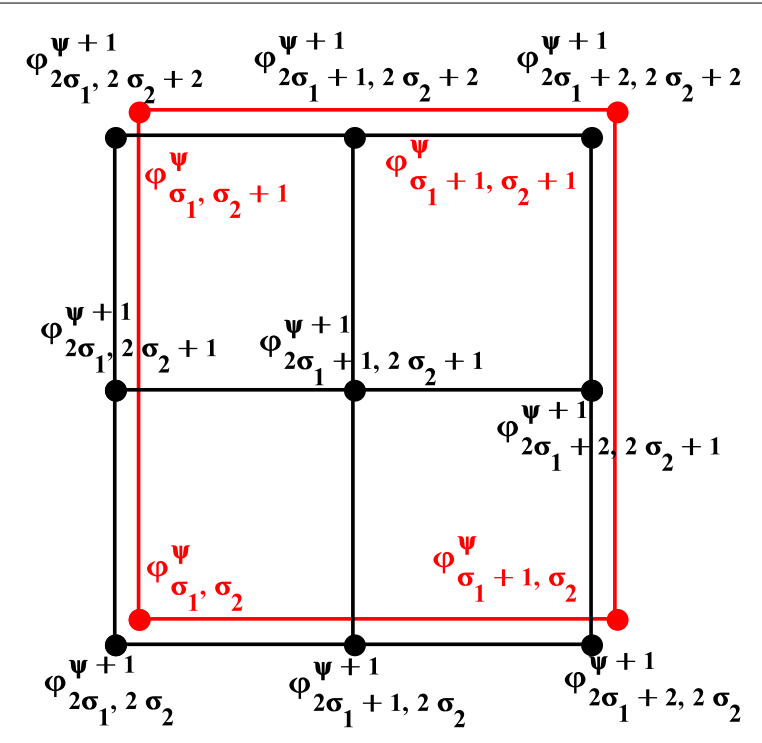

Figure 1 Subdivision of a surface patch by using GTPBRS

Illustration of the old points and the new points by GTPBRS is shown in Fig. 1. In this figure, red lines and red bullets represent mesh and points at the $\psi$ th refinement step, respectively, while black lines and black bullets represent the refined mesh and refined points by GTPBRS at the $(\psi+1)$ th refinement step, respectively.

Remark 1 The GTPBRS defined in (1) is the tensor product version of the two univariate schemes defined by

$$
\left\{\begin{array}{l}
\varphi_{2 \sigma_{1}}^{\psi+1}=\sum_{r=0}^{m} \rho_{r} \varphi_{\sigma_{1}+r}^{\psi} \\
\varphi_{2 \sigma_{1}+1}^{\psi+1}=\sum_{r=0}^{m} \chi_{r} \varphi_{\sigma_{1}+r}^{\psi}
\end{array}\right.
$$

and

$$
\left\{\begin{array}{l}
\varphi_{2 \sigma_{2}}^{\psi+1}=\sum_{s=0}^{m} \rho_{s} \varphi_{\sigma_{2}+s}^{\psi} \\
\varphi_{2 \sigma_{2}+1}^{\psi+1}=\sum_{s=0}^{m} \chi_{s} \varphi_{\sigma_{2}+s}^{\psi}
\end{array}\right.
$$

The mask of GTPBRS is obtained by taking tensor product of the masks of univariate schemes (3) and (4). That is, if $V=\left[\begin{array}{lllllll}\chi_{0} & \rho_{0} & \chi_{1} & \rho_{1} & \ldots & \chi_{m} & \rho_{m}\end{array}\right]$ is a one dimensional array containing the mask of the scheme (3) (this is also the mask of the scheme (4)), then the mask $\bar{V}$ of (1) is a two dimensional array obtained by

$$
\bar{V}=V \otimes V=V V^{T}
$$

\subsection{The first and second order GBDDRSs of GTPBRS}

In this subsection, we derive first and second order GBDDRSs of the GTPBRS. Firstly, we find the first and second order GBDDRSs for a fixed $m$ and then for an arbitrary $m$. 
Lemma 1 Let $\varphi^{\psi}=\left\{\left(\frac{\sigma_{1}}{2^{\psi}}, \frac{\sigma_{2}}{2^{\psi}}, \varphi_{\sigma_{1}, \sigma_{2}}^{\psi}\right): \sigma_{1}, \sigma_{2} \in \mathbb{Z}, \psi \in \mathbb{N}\right\}$ be a given mesh. If

$$
E_{\sigma_{1}, \sigma_{2}}^{\psi}=2^{2 \psi} \Delta_{\sigma_{1}} \Delta_{\sigma_{2}} \varphi_{\sigma_{1}, \sigma_{2}}^{\psi}
$$

is the first order divided difference, then the first order GBDDRS for a fixed $m=3$ is

$$
\left\{\begin{array}{l}
E_{2 \sigma_{1}, 2 \sigma_{2}}^{\psi+1}=2^{2} \sum_{l=0}^{2}\left(\Theta_{q 1} \sum_{t=0}^{2} \Theta_{u 1}\right), \\
E_{2 \sigma_{1}, 2 \sigma_{2}+1}^{\psi+1}=2^{2} \sum_{l=0}^{2}\left(\Theta_{q 1} \sum_{t=0}^{3} \Theta_{u 2}\right), \\
E_{2 \sigma_{1}+1,2 \sigma_{2}}^{\psi+1}=2^{2} \sum_{l=0}^{2}\left(\Theta_{q 1} \sum_{t=0}^{3} \Theta_{u 3}\right), \\
E_{2 \sigma_{1}+1,2 \sigma_{2}+1}^{\psi+1}=2^{2} \sum_{l=0}^{3}\left(\Theta_{q 2} \sum_{t=0}^{3} \Theta_{u 2}\right),
\end{array}\right.
$$

where $\Theta_{q 1}=\sum_{q=0}^{l}\left(\rho_{l-q}-\chi_{l-q}\right), \Theta_{q 2}=\sum_{q=0}^{l}\left(\chi_{l-q}-\rho_{l-q-1}\right), \Theta_{u 1}=\sum_{u=0}^{t}\left(\rho_{t-u}-\chi_{t-u}\right) E_{\sigma_{1}+l, \sigma_{2}+t}^{\psi}$, $\Theta_{u 2}=\sum_{u=0}^{t}\left(\chi_{t-u}-\rho_{t-u-1}\right) E_{\sigma_{1}+l, \sigma_{2}+t}^{\psi}$ and $\Theta_{u 3}=\sum_{u=0}^{t}\left(\chi_{t-u}-\rho_{t-u-1}\right) E_{\sigma_{1}+t, \sigma_{2}+l}^{\psi}$.

Proof If we replace $\sigma_{1}$ by $2 \sigma_{1}$ and $\sigma_{2}$ by $2 \sigma_{2}$ and $\psi$ by $\psi+1$ in (5), we obtain

$$
\begin{aligned}
E_{2 \sigma_{1}, 2 \sigma_{2}}^{\psi+1} & =2^{2(\psi+1)} \Delta_{\sigma_{1}} \Delta_{\sigma_{2}} \varphi_{2 \sigma_{1}, 2 \sigma_{2}}^{\psi+1} \\
& =2^{2(\psi+1)}\left(\varphi_{2 \sigma_{1}, 2 \sigma_{2}}^{\psi+1}-\varphi_{2 \sigma_{1}, 2 \sigma_{2}+1}^{\psi+1}-\varphi_{2 \sigma_{1}+1,2 \sigma_{2}}^{\psi+1}+\varphi_{2 \sigma_{1}+1,2 \sigma_{2}+1}^{\psi+1}\right) .
\end{aligned}
$$

Now by using (1) for $m=3$, we get

$$
\begin{aligned}
E_{2 \sigma_{1}, 2 \sigma_{2}}^{\psi+1} & \\
\quad & 2^{2(\psi+1)}\left[\left(\rho_{0} \rho_{0}-\chi_{0} \rho_{0}-\rho_{0} \chi_{0}+\chi_{0} \chi_{0}\right) \varphi_{\sigma_{1}, \sigma_{2}}^{\psi}+\left(\rho_{1} \rho_{0}-\chi_{1} \rho_{0}-\rho_{1} \chi_{0}+\chi_{1} \chi_{0}\right) \varphi_{\sigma_{1}+1, \sigma_{2}}^{\psi}\right. \\
& +\left(\rho_{2} \rho_{0}-\chi_{2} \rho_{0}-\rho_{2} \chi_{0}+\chi_{2} \chi_{0}\right) \varphi_{\sigma_{1}+2, \sigma_{2}}^{\psi}+\left(\rho_{3} \rho_{0}-\chi_{3} \rho_{0}-\rho_{3} \chi_{0}+\chi_{3} \chi_{0}\right) \varphi_{\sigma_{1}+3, \sigma_{2}}^{\psi} \\
& +\left(\rho_{0} \rho_{1}-\chi_{0} \rho_{1}-\rho_{0} \chi_{1}+\chi_{0} \chi_{1}\right) \varphi_{\sigma_{1}, \sigma_{2}+1}^{\psi}+\left(\rho_{1} \rho_{1}-\chi_{1} \rho_{1}-\rho_{1} \chi_{1}+\chi_{1} \chi_{1}\right) \varphi_{\sigma_{1}+1, \sigma_{2}+1}^{\psi} \\
& +\left(\rho_{2} \rho_{1}-\chi_{2} \rho_{1}-\rho_{2} \chi_{1}+\chi_{2} \chi_{1}\right) \varphi_{\sigma_{1}+2, \sigma_{2}+1}^{\psi}+\left(\rho_{3} \rho_{1}-\chi_{3} \rho_{1}-\rho_{3} \chi_{1}+\chi_{3} \chi_{1}\right) \varphi_{\sigma_{1}+3, \sigma_{2}+1}^{\psi} \\
& +\left(\rho_{0} \rho_{2}-\chi_{0} \rho_{2}-\rho_{0} \chi_{2}+\chi_{0} \chi_{2}\right) \varphi_{\sigma_{1}, \sigma_{2}+2}^{\psi}+\left(\rho_{1} \rho_{2}-\chi_{1} \rho_{2}-\rho_{1} \chi_{2}+\chi_{1} \chi_{2}\right) \varphi_{\sigma_{1}+1, \sigma_{2}+2}^{\psi} \\
& +\left(\rho_{2} \rho_{2}-\chi_{2} \rho_{2}-\rho_{2} \chi_{2}+\chi_{2} \chi_{2}\right) \varphi_{\sigma_{1}+2, \sigma_{2}+2}^{\psi}+\left(\rho_{3} \rho_{2}-\chi_{3} \rho_{2}-\rho_{3} \chi_{2}+\chi_{3} \chi_{2}\right) \varphi_{\sigma_{1}+3, \sigma_{2}+2}^{\psi} \\
& +\left(\rho_{0} \rho_{3}-\chi_{0} \rho_{3}-\rho_{0} \chi_{3}+\chi_{0} \chi_{3}\right) \varphi_{\sigma_{1}, \sigma_{2}+3}^{\psi}+\left(\rho_{1} \rho_{3}-\chi_{1} \rho_{3}-\rho_{1} \chi_{3}+\chi_{1} \chi_{3}\right) \varphi_{\sigma_{1}+1, \sigma_{2}+3}^{\psi} \\
& \left.+\left(\rho_{2} \rho_{3}-\chi_{2} \rho_{3}-\rho_{2} \chi_{3}+\chi_{2} \chi_{3}\right) \varphi_{\sigma_{1}+2, \sigma_{2}+3}^{\psi}+\left(\rho_{3} \rho_{3}-\chi_{3} \rho_{3}-\rho_{3} \chi_{3}+\chi_{3} \chi_{3}\right) \varphi_{\sigma_{1}+3, \sigma_{2}+3}^{\psi}\right] .
\end{aligned}
$$

After collecting the coefficients of $\varphi_{\sigma_{1}, \sigma_{2}}^{\psi}$, we get

$$
\begin{aligned}
E_{2 \sigma_{1}, 2 \sigma_{2}}^{\psi+1}= & 2^{2(\psi+1)}\left[\left\{\left(\rho_{0}-\chi_{0}\right)\left(\rho_{0}-\chi_{0}\right) \varphi_{\sigma_{1}, \sigma_{2}}^{\psi}+\left(\rho_{1}-\chi_{1}\right)\left(\rho_{0}-\chi_{0}\right) \varphi_{\sigma_{1}+1, \sigma_{2}}^{\psi}\right.\right. \\
& \left.+\left(\rho_{2}-\chi_{2}\right)\left(\rho_{0}-\chi_{0}\right) \varphi_{\sigma_{1}+2, \sigma_{2}}^{\psi}+\left(\rho_{3}-\chi_{3}\right)\left(\rho_{0}-\chi_{0}\right) \varphi_{\sigma_{1}+3, \sigma_{2}}^{\psi}\right\} \\
& +\left\{\left(\rho_{0}-\chi_{0}\right)\left(\rho_{1}-\chi_{1}\right) \varphi_{\sigma_{1}, \sigma_{2}+1}^{\psi}+\left(\rho_{1}-\chi_{1}\right)\left(\rho_{1}-\chi_{1}\right) \varphi_{\sigma_{1}+1, \sigma_{2}+1}^{\psi}\right. \\
& \left.+\left(\rho_{2}-\chi_{2}\right)\left(\rho_{1}-\chi_{1}\right) \varphi_{\sigma_{1}+2, \sigma_{2}+1}^{\psi}+\left(\rho_{3}-\chi_{3}\right)\left(\rho_{1}-\chi_{1}\right) \varphi_{\sigma_{1}+3, \sigma_{2}+1}^{\psi}\right\} \\
& +\left\{\left(\rho_{0}-\chi_{0}\right)\left(\rho_{2}-\chi_{2}\right) \varphi_{\sigma_{1}, \sigma_{2}+2}^{\psi}+\left(\rho_{1}-\chi_{1}\right)\left(\rho_{2}-\chi_{2}\right) \varphi_{\sigma_{1}+1, \sigma_{2}+2}^{\psi}\right. \\
& \left.+\left(\rho_{2}-\chi_{2}\right)\left(\rho_{2}-\chi_{2}\right) \varphi_{\sigma_{1}+2, \sigma_{2}+2}^{\psi}+\left(\rho_{3}-\chi_{3}\right)\left(\rho_{2}-\chi_{2}\right) \varphi_{\sigma_{1}+3, \sigma_{2}+2}^{\psi}\right\}
\end{aligned}
$$




$$
\begin{aligned}
& +\left\{\left(\rho_{0}-\chi_{0}\right)\left(\rho_{3}-\chi_{3}\right) \varphi_{\sigma_{1}, \sigma_{2}+3}^{\psi}+\left(\rho_{1}-\chi_{1}\right)\left(\rho_{3}-\chi_{3}\right) \varphi_{\sigma_{1}+1, \sigma_{2}+3}^{\psi}\right. \\
& \left.\left.+\left(\rho_{2}-\chi_{2}\right)\left(\rho_{3}-\chi_{3}\right) \varphi_{\sigma_{1}+2, \sigma_{2}+3}^{\psi}+\left(\rho_{3}-\chi_{3}\right)\left(\rho_{3}-\chi_{3}\right) \varphi_{\sigma_{1}+3, \sigma_{2}+3}^{\psi}\right\}\right] .
\end{aligned}
$$

We want to find a recursive relation in $E_{\sigma_{1}, \sigma_{2}}$ of the form

$$
\begin{aligned}
E_{2 \sigma_{1}, 2 \sigma_{2}}^{\psi+1}= & v_{00} E_{\sigma_{1}, \sigma_{2}}^{\psi}+v_{10} E_{\sigma_{1}+1, \sigma_{2}}^{\psi}+v_{20} E_{\sigma_{1}+2, \sigma_{2}}^{\psi}+v_{01} E_{\sigma_{1}, \sigma_{2}+1}^{\psi}+v_{11} E_{\sigma_{1}+1, \sigma_{2}+1}^{\psi} \\
& +v_{21} E_{\sigma_{1}+2, \sigma_{2}+1}^{\psi}+v_{02} E_{\sigma_{1}, \sigma_{2}+2}^{\psi}+v_{12} E_{\sigma_{1}+1, \sigma_{2}+2}^{\psi}+v_{22} E_{\sigma_{1}+2, \sigma_{2}+2}^{\psi} .
\end{aligned}
$$

If we use the first order divided differences in (8), we get

$$
\begin{aligned}
E_{2 \sigma_{1}, 2 \sigma_{2}}^{\psi+1}= & 2^{2 \psi}\left[\left\{v_{00} \varphi_{\sigma_{1}, \sigma_{2}}^{\psi}+\left(-v_{00}+v_{01}\right) \varphi_{\sigma_{1}, \sigma_{2}+1}^{\psi}+\left(-v_{01}+v_{02}\right) \varphi_{\sigma_{1}, \sigma_{2}+2}^{\psi}-v_{20} \varphi_{\sigma_{1}+3, \sigma_{2}}^{\psi}\right\}\right. \\
& +\left\{\left(-v_{00}+v_{10}\right) \varphi_{\sigma_{1}+1, \sigma_{2}}^{\psi}+\left(v_{00}-v_{01}-v_{10}+v_{11}\right) \varphi_{\sigma_{1}+1, \sigma_{2}+1}^{\psi}+\left(v_{01}-v_{02}\right.\right. \\
& \left.\left.-v_{11}+v_{12}\right) \varphi_{\sigma_{1}+1, \sigma_{2}+2}^{\psi}+\left(v_{02}-v_{12}\right) \varphi_{\sigma_{1}+3, \sigma_{2}+1}^{\psi}\right\}+\left\{\left(-v_{10}+v_{20}\right) \varphi_{\sigma_{1}+2, \sigma_{2}}^{\psi}\right. \\
& +\left(v_{10}-v_{11}-v_{20}+v_{21}\right) \varphi_{\sigma_{1}+2, \sigma_{2}+1}^{\psi}+\left(v_{11}-v_{12}-v_{21}+v_{22}\right) \varphi_{\sigma_{1}+2, \sigma_{2}+2}^{\psi} \\
& \left.+\left(v_{12}-v_{22}\right) \varphi_{\sigma_{1}+2, \sigma_{2}+3}^{\psi}\right\}+\left\{-v_{20} \varphi_{\sigma_{1}+3, \sigma_{2}}^{\psi}+\left(v_{20}-v_{21}\right) \varphi_{\sigma_{1}+3, \sigma_{2}+1}^{\psi}+\left(v_{21}\right.\right. \\
& \left.\left.\left.-v_{22}\right) \varphi_{\sigma_{1}+3, \sigma_{2}+2}^{\psi}+v_{22} \varphi_{\sigma_{1}+3, \sigma_{2}+3}^{\psi}\right\}\right] .
\end{aligned}
$$

By comparing the coefficients of the $\varphi_{\sigma_{1}, \sigma_{2}}^{\psi}$ in (7) and (9), we get

$$
\begin{aligned}
& v_{00}=2^{2}\left(\rho_{0}-\chi_{0}\right)\left(\rho_{0}-\chi_{0}\right), \\
& v_{10}=2^{2}\left(\rho_{0}-\chi_{0}\right)\left(\rho_{1}+\rho_{0}-\chi_{1}-\chi_{0}\right), \\
& v_{20}=2^{2}\left(\rho_{0}-\chi_{0}\right)\left(\rho_{2}+\rho_{1}+\rho_{0}-\chi_{2}-\chi_{1}-\chi_{0}\right), \\
& v_{01}=2^{2}\left(\rho_{1}+\rho_{0}-\chi_{1}-\chi_{0}\right)\left(\rho_{0}-\chi_{0}\right), \\
& v_{11}=2^{2}\left(\rho_{1}+\rho_{0}-\chi_{1}-\chi_{0}\right)\left(\rho_{1}+\rho_{0}-\chi_{1}-\chi_{0}\right), \\
& v_{21}=2^{2}\left(\rho_{1}+\rho_{0}-\chi_{1}-\chi_{0}\right)\left(\rho_{2}+\rho_{1}+\rho_{0}-\chi_{2}-\chi_{1}-\chi_{0}\right), \\
& v_{02}=2^{2}\left(\rho_{2}+\rho_{1}+\rho_{0}-\chi_{2}-\chi_{1}-\chi_{0}\right)\left(\rho_{0}-\chi_{0}\right), \\
& v_{12}=2^{2}\left(\rho_{2}+\rho_{1}+\rho_{0}-\chi_{2}-\chi_{1}-\chi_{0}\right)\left(\rho_{1}+\rho_{0}-\chi_{1}-\chi_{0}\right), \\
& v_{22}=2^{2}\left(\rho_{2}+\rho_{1}+\rho_{0}-\chi_{2}-\chi_{1}-\chi_{0}\right)\left(\rho_{2}+\rho_{1}+\rho_{0}-\chi_{2}-\chi_{1}-\chi_{0}\right) .
\end{aligned}
$$

By substituting these values in (8), we get

$$
\begin{aligned}
E_{2 \sigma_{1}, 2 \sigma_{2}}^{\psi+1}= & 2^{2}\left[( \rho _ { 0 } - \chi _ { 0 } ) \left\{\left(\rho_{0}-\chi_{0}\right) E_{\sigma_{1}, \sigma_{2}}^{\psi}+\left(\rho_{1}+\rho_{0}-\chi_{1}-\chi_{0}\right) E_{\sigma_{1}+1, \sigma_{2}}^{\psi}+\left(\rho_{2}+\rho_{1}+\rho_{0}-\chi_{2}\right.\right.\right. \\
& \left.\left.-\chi_{1}-\chi_{0}\right) E_{\sigma_{1}+2, \sigma_{2}}^{\psi}\right\}+\left(\rho_{1}+\rho_{0}-\chi_{1}-\chi_{0}\right)\left\{\left(\rho_{0}-\chi_{0}\right) E_{\sigma_{1}, \sigma_{2}+1}^{\psi}+\left(\rho_{1}+\rho_{0}-\chi_{1}\right.\right. \\
& \left.\left.-\chi_{0}\right) E_{\sigma_{1}+1, \sigma_{2}+1}^{\psi}+\left(\rho_{2}+\rho_{1}+\rho_{0}-\chi_{2}-\chi_{1}-\chi_{0}\right) E_{\sigma_{1}+2, \sigma_{2}+1}^{\psi}\right\}+\left(\rho_{2}+\rho_{1}+\rho_{0}-\chi_{2}\right. \\
& \left.-\chi_{1}-\chi_{0}\right)\left\{\left(\rho_{0}-\chi_{0}\right) E_{\sigma_{1}, \sigma_{2}+2}^{\psi}+\left(\rho_{1}+\rho_{0}-\chi_{1}-\chi_{0}\right) E_{\sigma_{1}+1, \sigma_{2}+2}^{\psi}\right. \\
& \left.\left.+\left(\rho_{2}+\rho_{1}+\rho_{0}-\chi_{2}-\chi_{1}-\chi_{0}\right) E_{\sigma_{1}+2, \sigma_{2}+2}^{\psi}\right\}\right] .
\end{aligned}
$$


By simplifying, we obtain the following first refinement rule of the first order GBDDRS:

$$
E_{2 \sigma_{1}, 2 \sigma_{2}}^{\psi+1}=2^{2} \sum_{l=0}^{2}\left[\sum_{q=0}^{l}\left(\rho_{l-q}-\chi_{l-q}\right) \sum_{t=0}^{2}\left\{\sum_{u=0}^{t}\left(\rho_{t-u}-\chi_{t-u}\right) E_{\sigma_{1}+l, \sigma_{2}+t}^{\psi}\right\}\right] .
$$

Now, we calculate the second refinement rule of the first order GBDDRS. Therefore, we replace $\sigma_{1}$ by $2 \sigma_{1}, \sigma_{2}$ by $2 \sigma_{2}+1$ and $\psi$ by $\psi+1$ in (5) and obtain

$$
E_{2 \sigma_{1}, 2 \sigma_{2}+1}^{\psi+1}=2^{2(\psi+1)} \Delta_{\sigma_{1}} \Delta_{\sigma_{2}} \varphi_{2 \sigma_{1}, 2 \sigma_{2}+1}^{\psi+1}
$$

By adopting the procedure above, we get the following second refinement rule of the first order GBDDRS:

$$
E_{2 \sigma_{1}, 2 \sigma_{2}+1}^{\psi+1}=2^{2} \sum_{l=0}^{2}\left[\sum_{q=0}^{l}\left(\rho_{l-q}-\chi_{l-q}\right) \sum_{t=0}^{3}\left\{\sum_{u=0}^{t}\left(\chi_{t-u}-\rho_{t-u-1}\right) E_{\sigma_{1}+l, \sigma_{2}+t}^{\psi}\right\}\right] .
$$

Similarly, the third and fourth refinement rules of the first order GBDDRS can be obtained. Hence the lemma is proved.

From Lemma 1, the following result can be obtained.

Lemma 2 Let $\varphi^{\psi}=\left\{\left(\frac{\sigma_{1}}{2^{\psi}}, \frac{\sigma_{2}}{2^{\psi}}, \varphi_{\sigma_{1}, \sigma_{2}}^{\psi}\right): \sigma_{1}, \sigma_{2} \in \mathbb{Z}, \psi \in \mathbb{N}\right\}$ be a given mesh and

$$
E_{\sigma_{1}, \sigma_{2}}^{\psi}=2^{2 \psi} \Delta_{\sigma_{1}} \Delta_{\sigma_{2}} \varphi_{\sigma_{1}, \sigma_{2}}^{\psi}
$$

be the first order divided differences, then the first order GBDDRS for an arbitrary $m$ is

$$
\left\{\begin{array}{l}
E_{2 \sigma_{1}, 2 \sigma_{2}}^{\psi+1}=2^{2} \sum_{l=0}^{m-1}\left(\Theta_{q 1} \sum_{t=0}^{m-1} \Theta_{u 1}\right), \\
E_{2 \sigma_{1}, 2 \sigma_{2}+1}^{\psi+1}=2^{2} \sum_{l=0}^{m-1}\left(\Theta_{q 1} \sum_{t=0}^{m} \Theta_{u 2}\right), \\
E_{2 \sigma_{1}+1,2 \sigma_{2}}^{\psi+1}=2^{2} \sum_{l=0}^{m-1}\left(\Theta_{q 1} \sum_{t=0}^{m} \Theta_{u 3}\right), \\
E_{2 \sigma_{1}+1,2 \sigma_{2}+1}^{\psi+1}=2^{2} \sum_{l=0}^{m}\left(\Theta_{q 2} \sum_{t=0}^{m} \Theta_{u 2}\right),
\end{array}\right.
$$

where $\Theta_{q 1}=\sum_{q=0}^{l}\left(\rho_{l-q}-\chi_{l-q}\right), \Theta_{q 2}=\sum_{q=0}^{l}\left(\chi_{l-q}-\rho_{l-q-1}\right), \Theta_{u 1}=\sum_{u=0}^{t}\left(\rho_{t-u}-\chi_{t-u}\right) E_{\sigma_{1}+l, \sigma_{2}+t}^{\psi}$, $\Theta_{u 2}=\sum_{u=0}^{t}\left(\chi_{t-u}-\rho_{t-u-1}\right) E_{\sigma_{1}+l, \sigma_{2}+t}^{\psi}$ and $\Theta_{u 3}=\sum_{u=0}^{t}\left(\chi_{t-u}-\rho_{t-u-1}\right) E_{\sigma_{1}+t, \sigma_{2}+l}^{\psi}$.

Now we calculate second order GBDDRS of the GTPBRS.

Lemma 3 Let $\varphi^{\psi}=\left\{\left(\frac{\sigma_{1}}{2^{\psi}}, \frac{\sigma_{2}}{2^{\psi}}, \varphi_{\sigma_{1}, \sigma_{2}}^{\psi}\right): \sigma_{1}, \sigma_{2} \in \mathbb{Z}, \psi \in \mathbb{N}\right\}$ be a given mesh and

$$
H_{\sigma_{1}, \sigma_{2}}^{\psi}=2^{4 \psi}(2 !)^{-2} \Delta_{\sigma_{1}}^{2} \Delta_{\sigma_{2}}^{2} \varphi_{\sigma_{1}, \sigma_{2}}^{\psi}, \quad \forall \sigma_{1}, \sigma_{2} \in \mathbb{Z}
$$


be the second order divided differences, then the second order GBDDRS for a fixed $m=3$ is

$$
\left\{\begin{aligned}
H_{2 \sigma_{1}, 2 \sigma_{2}}^{\psi+1}= & 2^{4} \sum_{l=0}^{2}\left[\sum_{q=0}^{l}\left\{A_{q 1} \rho_{l-q}-A_{q 2} \chi_{l-q}\right\}\right. \\
& \left.\times \sum_{t=0}^{2}\left\{\sum_{u=0}^{t}\left\{A_{u 1} \rho_{t-u}-A_{u 2} \chi_{t-u}\right\} H_{\sigma_{1}+l, \sigma_{2}+t}^{\psi}\right\}\right] \\
H_{2 \sigma_{1}+1,2 \sigma_{2}}^{\psi+1}= & 2^{4} \sum_{l=0}^{2}\left[\sum_{q=0}^{l}\left\{A_{q 1} \rho_{l-q}-A_{q 2} \chi_{l-q}\right\}\right. \\
& \left.\times \sum_{t=0}^{2}\left\{\sum_{u=0}^{t}\left\{A_{u 1} \chi_{t-u}-A_{u 0} \rho_{t-u}\right\} H_{\sigma_{1}+t, \sigma_{2}+l}^{\psi}\right\}\right] \\
H_{2 \sigma_{1}, 2 \sigma_{2}+1}^{\psi+1}= & 2^{4} \sum_{l=0}^{2}\left[\sum_{q=0}^{l}\left\{A_{q 1} \rho_{l-q}-A_{q 2} \chi_{l-q}\right\}\right. \\
& \left.\times \sum_{t=0}^{2}\left\{\sum_{u=0}^{t}\left\{A_{u 1} \chi_{t-u}-A_{u 0} \rho_{t-u}\right\} H_{\sigma_{1}+l, \sigma_{2}+t}^{\psi}\right\}\right] \\
H_{2 \sigma_{1}+1,2 \sigma_{2}+1}^{\psi+1}= & 2^{4} \sum_{l=0}^{2}\left[\sum_{q=0}^{l}\left\{A_{q 1} \chi_{l-q}-A_{q 0} \rho_{l-q}\right\}\right. \\
& \left.\times \sum_{t=0}^{2}\left\{\sum_{u=0}^{t}\left\{A_{u 1} \chi_{t-u}-A_{u 0} \rho_{t-u}\right\} H_{\sigma_{1}+l, \sigma_{2}+t}^{\psi}\right\}\right]
\end{aligned}\right.
$$

where $A_{q 0}=2 q, A_{q 1}=2 q+1, A_{q 2}=2 q+2, A_{u 0}=2 u, A_{u 1}=2 u+1$ and $A_{u 2}=2 u+2$.

Proof If we replace $\sigma_{1}$ by $2 \sigma_{1}, \sigma_{2}$ by $2 \sigma_{2}$ and $\psi$ by $\psi+1$ in (11), we obtain

$$
\begin{aligned}
H_{2 \sigma_{1}, 2 \sigma_{2}}^{\psi+1}= & 2^{4(\psi+1)} 2^{-2} \Delta_{\sigma_{1}}^{2} \Delta_{\sigma_{2}}^{2} \varphi_{2 \sigma_{1}, 2 \sigma_{2}}^{\psi+1}=2^{4(\psi+1)} 2^{-2}\left(\varphi_{2 \sigma_{1}, 2 \sigma_{2}}^{\psi+1}-2 \varphi_{2 \sigma_{1}+1,2 \sigma_{2}}^{\psi+1}+\varphi_{2 \sigma_{1}+2,2 \sigma_{2}}^{\psi+1}\right. \\
& -2 \varphi_{2 \sigma_{1}, 2 \sigma_{2}+1}^{\psi+1}+4 \varphi_{2 \sigma_{1}+1,2 \sigma_{2}+1}^{\psi+1}-2 \varphi_{2 \sigma_{1}+2,2 \sigma_{2}+1}^{\psi+1}+\varphi_{2 \sigma_{1}, 2 \sigma_{2}+2}^{\psi+1}-2 \varphi_{2 \sigma_{1}+1,2 \sigma_{2}+2}^{\psi+1} \\
& \left.+\varphi_{2 \sigma_{1}+2,2 \sigma_{2}+2}^{\psi+1}\right) .
\end{aligned}
$$

Now by using (1) for $m=3$, we obtain

$$
\begin{aligned}
H_{2 \sigma_{1}, 2 \sigma_{2}}^{\psi+1}= & 2^{4(\psi+1)} 2^{-2}\left[( \rho _ { 0 } - 2 \chi _ { 0 } ) \left\{\left(\rho_{0}-2 \chi_{0}\right) \varphi_{\sigma_{1}, \sigma_{2}}^{\psi}+\left(\rho_{1}+\rho_{0}-2 \chi_{1}\right) \varphi_{\sigma_{1}, \sigma_{2}+1}^{\psi}\right.\right. \\
& \left.+\left(\rho_{2}+\rho_{1}-2 \chi_{2}\right) \varphi_{\sigma_{1}, \sigma_{2}+2}^{\psi}+\left(\rho_{3}+\rho_{2}-2 \chi_{3}\right) \varphi_{\sigma_{1}, \sigma_{2}+3}^{\psi}+\rho_{3} \varphi_{\sigma_{1}, \sigma_{2}+4}^{\psi}\right\} \\
& +\left(\rho_{1}+\rho_{0}-2 \chi_{1}\right)\left\{\left(\rho_{0}-2 \chi_{0}\right) \varphi_{\sigma_{1}+1, \sigma_{2}}^{\psi}+\left(\rho_{1}+\rho_{0}-2 \chi_{1}\right) \varphi_{\sigma_{1}+1, \sigma_{2}+1}^{\psi}\right. \\
& \left.+\left(\rho_{2}+\rho_{1}-2 \chi_{2}\right) \varphi_{\sigma_{1}+1, \sigma_{2}+2}^{\psi}+\left(\rho_{3}+\rho_{2}-2 \chi_{3}\right) \varphi_{\sigma_{1}+1, \sigma_{2}+3}^{\psi}+\rho_{3} \varphi_{\sigma_{1}+1, \sigma_{2}+4}^{\psi}\right\} \\
& +\left(\rho_{2}+\rho_{1}-2 \chi_{2}\right)\left\{\left(\rho_{0}-2 \chi_{0}\right) \varphi_{\sigma_{1}+2, \sigma_{2}}^{\psi}+\left(\rho_{1}+\rho_{0}-2 \chi_{1}\right) \varphi_{\sigma_{1}+2, \sigma_{2}+1}^{\psi}\right. \\
& \left.+\left(\rho_{2}+\rho_{1}-2 \chi_{2}\right) \varphi_{\sigma_{1}+2, \sigma_{2}+2}^{\psi}+\left(\rho_{3}+\rho_{2}-2 \chi_{3}\right) \varphi_{\sigma_{1}+2, \sigma_{2}+3}^{\psi}+\rho_{3} \varphi_{\sigma_{1}+2, \sigma_{2}+4}^{\psi}\right\} \\
& +\left(\rho_{3}+\rho_{2}-2 \chi_{3}\right)\left\{\left(\rho_{0}-2 \chi_{0}\right) \varphi_{\sigma_{1}+3, \sigma_{2}}^{\psi}+\left(\rho_{1}+\rho_{0}-2 \chi_{1}\right) \varphi_{\sigma_{1}+3, \sigma_{2}+1}^{\psi}\right. \\
& \left.+\left(\rho_{2}+\rho_{1}-2 \chi_{2}\right) \varphi_{\sigma_{1}+3, \sigma_{2}+2}^{\psi}+\left(\rho_{3}+\rho_{2}-2 \chi_{3}\right) \varphi_{\sigma_{1}+3, \sigma_{2}+3}^{\psi}+\rho_{3} \varphi_{\sigma_{1}+3, \sigma_{2}+4}^{\psi}\right\} \\
& +\rho_{3}\left\{\left(\rho_{0}-2 \chi_{0}\right) \varphi_{\sigma_{1}+4, \sigma_{2}}^{\psi}+\left(\rho_{1}+\rho_{0}-2 \chi_{1}\right) \varphi_{\sigma_{1}+4, \sigma_{2}+1}^{\psi}+\left(\rho_{2}+\rho_{1}-2 \chi_{2}\right)\right. \\
& \left.\left.\times \varphi_{\sigma_{1}+4, \sigma_{2}+2}^{\psi}+\left(\rho_{3}+\rho_{2}-2 \chi_{3}\right) \varphi_{\sigma_{1}+4, \sigma_{2}+3}^{\psi}+\rho_{3} \varphi_{\sigma_{1}+4, \sigma_{2}+4}^{\psi}\right\}\right] .
\end{aligned}
$$

We want to find the refinement equation of the second order GBDDRS. Therefore, we are seeking an equation of the following type:

$$
\begin{aligned}
H_{2 \sigma_{1}, 2 \sigma_{2}}^{\psi+1}= & v_{00} H_{\sigma_{1}, \sigma_{2}}^{\psi}+v_{10} H_{\sigma_{1}+1, \sigma_{2}}^{\psi}+v_{20} H_{\sigma_{1}+2, \sigma_{2}}^{\psi}+v_{01} H_{\sigma_{1}, \sigma_{2}+1}^{\psi}+v_{11} H_{\sigma_{1}+1, \sigma_{2}+1}^{\psi} \\
& +v_{21} H_{\sigma_{1}+2, \sigma_{2}+1}^{\psi}+v_{02} H_{\sigma_{1}, \sigma_{2}+2}^{\psi}+v_{12} H_{\sigma_{1}+1, \sigma_{2}+2}^{\psi}+v_{22} H_{\sigma_{1}+2, \sigma_{2}+2}^{\psi} .
\end{aligned}
$$

By substituting values and simplifying, we get

$$
H_{2 \sigma_{1}, 2 \sigma_{2}}^{\psi+1}=2^{4 \psi} 2^{-2}\left[v_{00} \varphi_{\sigma_{1}, \sigma_{2}}^{\psi}+\left(-2 v_{00}+v_{01}\right) \varphi_{\sigma_{1}, \sigma_{2}+1}^{\psi}+\left(v_{00}-2 v_{01}+v_{02}\right) \varphi_{\sigma_{1}, \sigma_{2}+2}^{\psi}\right.
$$




$$
\begin{aligned}
& +\left(v_{01}-2 v_{02}\right) \varphi_{\sigma_{1}, \sigma_{2}+3}^{\psi}+v_{02} \varphi_{\sigma_{1}, \sigma_{2}+4}^{\psi}+\left(-2 v_{00}+v_{10}\right) \varphi_{\sigma_{1}+1, \sigma_{2}}^{\psi}+\left(4 v_{00}\right. \\
& \left.-2 v_{10}-2 v_{01}+v_{11}\right) \varphi_{\sigma_{1}+1, \sigma_{2}+1}^{\psi}+\left(-2 v_{00}+v_{10}+4 v_{01}-2 v_{11}-2 v_{02}\right. \\
& \left.+v_{12}\right) \varphi_{\sigma_{1}+1, \sigma_{2}+2}^{\psi}+\left(-2 v_{01}+v_{11}+4 v_{02}-2 v_{12}\right) \varphi_{\sigma_{1}+1, \sigma_{2}+3}^{\psi}+\left(-2 v_{02}\right. \\
& \left.+v_{12}\right) \varphi_{\sigma_{1}+1, \sigma_{2}+4}^{\psi}+\left(v_{00}-2 v_{10}+v_{20}\right) \varphi_{\sigma_{1}+2, \sigma_{2}}^{\psi}+\left(-2 v_{00}+4 v_{10}-2 v_{20}\right. \\
& \left.+v_{01}-2 v_{11}+v_{21}\right) \varphi_{\sigma_{1}+2, \sigma_{2}+1}^{\psi}+\left(v_{00}-2 v_{10}+v_{20}-2 v_{01}+4 v_{11}-2\right. \\
& \left.\times v_{21}+v_{02}-2 v_{12}+v_{22}\right) \varphi_{\sigma_{1}+2, \sigma_{2}+2}^{\psi}+\left(v_{01}-2 v_{11}+v_{21}-2 v_{02}+4\right. \\
& \left.\times v_{12}-2 v_{22}\right) \varphi_{\sigma_{1}+2, \sigma_{2}+3}^{\psi}+\left(v_{02}-2 v_{12}+v_{22}\right) \varphi_{\sigma_{1}+2, \sigma_{2}+4}^{\psi}+\left(v_{10}-2 v_{20}\right) \\
& \times \varphi_{\sigma_{1}+3, \sigma_{2}}^{\psi}+\left(-2 v_{10}+4 v_{20}+v_{11}-2 v_{21}\right) \varphi_{\sigma_{1}+3, \sigma_{2}+1}^{\psi}+\left(v_{10}-2 v_{20}-2\right. \\
& \left.\times v_{11}+4 v_{21}+v_{12}-2 v_{22}\right) \varphi_{\sigma_{1}+3, \sigma_{2}+2}^{\psi}+\left(v_{11}-2 v_{21}-2 v_{12}+4 v_{22}\right) \\
& \times \varphi_{\sigma_{1}+3, \sigma_{2}+3}^{\psi}+\left(v_{12}-2 v_{22}\right) \varphi_{\sigma_{1}+3, \sigma_{2}+4}^{\psi}+v_{20} \varphi_{\sigma_{1}+4, \sigma_{2}}^{\psi}+\left(-2 v_{20}+v_{21}\right) \varphi_{\sigma_{1}+4, \sigma_{2}+1}^{\psi} \\
& \left.+\left(v_{20}-2 v_{21}+v_{22}\right) \varphi_{\sigma_{1}+4, \sigma_{2}+2}^{\psi}+\left(v_{21}-2 v_{22}\right) \varphi_{\sigma_{1}+4, \sigma_{2}+3}^{\psi}+v_{22} \varphi_{\sigma_{1}+4, \sigma_{2}+4}^{\psi}\right]
\end{aligned}
$$

Comparing coefficients of the $\varphi_{\sigma_{1}, \sigma_{2}}^{\psi}$ in (13) and (15), we get

$$
\begin{aligned}
& v_{00}=2^{4}\left(\rho_{0}-2 \chi_{0}\right)\left(\rho_{0}-2 \chi_{0}\right), \\
& v_{10}=2^{4}\left(\rho_{0}-2 \chi_{0}\right)\left(\rho_{1}+3 \rho_{0}-2 \chi_{1}-4 \chi_{0}\right), \\
& v_{20}=2^{4}\left(\rho_{0}-2 \chi_{0}\right)\left(\rho_{2}+3 \rho_{1}+5 \rho_{0}-2 \chi_{2}-4 \chi_{1}-6 \chi_{0}\right), \\
& v_{01}=2^{4}\left(\rho_{1}+3 \rho_{0}-2 \chi_{1}-4 \chi_{0}\right)\left(\rho_{0}-2 \chi_{0}\right), \\
& v_{11}=2^{4}\left(\rho_{1}+3 \rho_{0}-2 \chi_{1}-4 \chi_{0}\right)\left(\rho_{1}+3 \rho_{0}-2 \chi_{1}-4 \chi_{0}\right), \\
& v_{21}=2^{4}\left(\rho_{1}+3 \rho_{0}-2 \chi_{1}-4 \chi_{0}\right)\left(\rho_{2}+3 \rho_{1}+5 \rho_{0}-2 \chi_{2}-4 \chi_{1}-6 \chi_{0}\right), \\
& v_{02}=2^{4}\left(\rho_{2}+3 \rho_{1}+5 \rho_{0}-2 \chi_{2}-4 \chi_{1}-6 \chi_{0}\right)\left(\rho_{0}-2 \chi_{0}\right), \\
& v_{12}=2^{4}\left(\rho_{2}+3 \rho_{1}+5 \rho_{0}-2 \chi_{2}-4 \chi_{1}-6 \chi_{0}\right)\left(\rho_{1}+3 \rho_{0}-2 \chi_{1}-4 \chi_{0}\right), \\
& v_{22}=2^{4}\left(\rho_{2}+3 \rho_{1}+5 \rho_{0}-2 \chi_{2}-4 \chi_{1}-6 \chi_{0}\right)\left(\rho_{2}+3 \rho_{1}+5 \rho_{0}-2 \chi_{2}-4 \chi_{1}-6 \chi_{0}\right) .
\end{aligned}
$$

Now we substitute all the above values in (14), we get the first refinement rule of the second order GBDDRS, i.e.

$$
\begin{aligned}
H_{2 \sigma_{1}, 2 \sigma_{2}}^{\psi+1}= & 2^{4} \sum_{l=0}^{2}\left[\sum_{q=0}^{l}\left\{(2 q+1) \rho_{l-q}-(2 q+2) \chi_{l-q}\right\}\right. \\
& \left.\times \sum_{t=0}^{2}\left\{\sum_{u=0}^{t}\left\{(2 u+1) \rho_{t-u}-(2 u+2) \chi_{t-u}\right\} H_{\sigma_{1}+l, \sigma_{2}+t}^{\psi}\right\}\right] .
\end{aligned}
$$

By adopting the same procedure as above, we find the following second refinement rule of the second order GBDDRS:

$$
\begin{aligned}
H_{2 \sigma_{1}+1,2 \sigma_{2}}^{\psi+1}= & 2^{4} \sum_{l=0}^{2}\left[\sum_{q=0}^{l}\left\{(2 q+1) \rho_{l-q}-(2 q+2) \chi_{l-q}\right\}\right. \\
& \left.\times \sum_{t=0}^{2}\left\{\sum_{u=0}^{t}\left\{(2 u+1) \chi_{t-u}-2 u \rho_{t-u}\right\} H_{\sigma_{1}+t, \sigma_{2}+l}^{\psi}\right\}\right] .
\end{aligned}
$$


Similarly, the third and fourth refinement rules of the second order GBDDRS can be obtained. Hence our result proved.

Lemma 4 Let $\varphi^{\psi}=\left\{\left(\frac{\sigma_{1}}{2 \psi}, \frac{\sigma_{2}}{2 \psi}, \varphi_{\sigma_{1}, \sigma_{2}}^{\psi}\right): \sigma_{1}, \sigma_{2} \in \mathbb{Z}, \psi \in \mathbb{N}\right\}$ be a given mesh and

$$
H_{\sigma_{1}, \sigma_{2}}^{\psi}=2^{4 \psi}(2 !)^{-2} \Delta_{\sigma_{1}}^{2} \Delta_{\sigma_{2}}^{2} \varphi_{\sigma_{1}, \sigma_{2}}^{\psi}, \quad \forall \sigma_{1}, \sigma_{2} \in \mathbb{Z}
$$

be the second order divided differences, then the second order GBDDRS for an arbitrary $m$ is

$$
\left\{\begin{aligned}
H_{2 \sigma_{1}, 2 \sigma_{2}}^{\psi+1}= & 2^{4} \sum_{l=0}^{m-1}\left[\sum_{q=0}^{l}\left\{A_{q 1} \rho_{l-q}-A_{q 2} \chi_{l-q}\right\}\right. \\
& \left.\times \sum_{t=0}^{m-1}\left\{\sum_{u=0}^{t}\left\{A_{u 1} \rho_{t-u}-A_{u 2} \chi_{t-u}\right\} H_{\sigma_{1}+l, \sigma_{2}+t}^{\psi}\right\}\right], \\
H_{2 \sigma_{1}+1,2 \sigma_{2}}^{\psi+1}= & 2^{4} \sum_{l=0}^{m-1}\left[\sum_{q=0}^{l}\left\{A_{q 1} \rho_{l-q}-A_{q 2} \chi_{l-q}\right\}\right. \\
& \left.\times \sum_{t=0}^{m-1}\left\{\sum_{u=0}^{t}\left\{A_{u 1} \chi_{t-u}-A_{u 0} \rho_{t-u}\right\} H_{\sigma_{1}+t, \sigma_{2}+l}^{\psi}\right\}\right], \\
H_{2 \sigma_{1}, 2 \sigma_{2}+1}^{\psi+1}= & 2^{4} \sum_{l=0}^{m-1}\left[\sum_{q=0}^{l}\left\{A_{q 1} \rho_{l-q}-A_{q 2} \chi_{l-q}\right\}\right. \\
& \left.\times \sum_{t=0}^{m-1}\left\{\sum_{u=0}^{t}\left\{A_{u 1} \chi_{t-u}-A_{u 0} \rho_{t-u}\right\} H_{\sigma_{1+l, \sigma_{2}+t}}^{\psi}\right\}\right], \\
H_{2 \sigma_{1}+1,2 \sigma_{2}+1}^{\psi+1}= & 2^{4} \sum_{l=0}^{m-1}\left[\sum_{q=0}^{l}\left\{A_{q 1} \chi_{l-q}-A_{q 0} \rho_{l-q}\right\}\right. \\
& \left.\times \sum_{t=0}^{m-1}\left\{\sum_{u=0}^{t}\left\{A_{u 1} \chi_{t-u}-A_{u 0} \rho_{t-u}\right\} H_{\sigma_{1}+l, \sigma_{2}+t}^{\psi}\right\}\right],
\end{aligned}\right.
$$

where $A_{q 0}=2 q, A_{q 1}=2 q+1, A_{q 2}=2 q+2, A_{u 0}=2 u, A_{u 1}=2 u+1$ and $A_{u 2}=2 u+2$.

\subsection{Convergence of the bivariate binary and its GBDDRS}

In this subsection, firstly we calculate the derivatives of two Bernstein polynomials in $r$ and $t$. Secondly, we take the tensor product of these derivatives. Lastly, we prove that the result converge uniformly to some continuous function. Throughout the article, $\Gamma_{[0, n]^{2}}$ denotes the set of continuous functions on the closed and bounded interval $[0, n] \times[0, n]$.

Lemma 5 Let $\varphi^{\psi}=\left\{\left(\frac{\sigma_{1}}{2^{\psi}}, \frac{\sigma_{2}}{2^{\psi}}, \varphi_{\sigma_{1}, \sigma_{2}}^{\psi}\right): \sigma_{1}, \sigma_{2} \in \mathbb{Z}, \psi \in \mathbb{N}\right\}$ be a mesh produced by the GTP$B R S$ at the $\psi$ th refinement level and $E^{\psi}=\left\{\left(\frac{\sigma_{1}}{2 \psi}, \frac{\sigma_{2}}{2 \psi}, E_{\sigma_{1}, \sigma_{2}}^{\psi}\right): \sigma_{1}, \sigma_{2} \in \mathbb{Z}, \psi \in \mathbb{N}\right\}$ be a mesh produced by the first order GBDDRS at the $\psi$ th refinement level. If $\lim _{\psi \rightarrow \infty} \varphi^{\psi}=\varphi \in \Gamma_{[0, n]^{2}}$, and $\varphi$ is the limiting model produced by GTPBRS then $E=\varphi^{\prime}$.

Proof Bernstein polynomials for $r, t \in[0, n]$ are

$$
\left\{\begin{array}{l}
b_{\psi_{1}}(r)=\sum_{\sigma_{1}=0}^{N}\left(\begin{array}{c}
N \\
\sigma_{1}
\end{array}\right)\left(\frac{r}{n}\right)^{\sigma_{1}}\left(1-\frac{r}{n}\right)^{N-\sigma_{1}}, \\
b_{\psi_{2}}(t)=\sum_{\sigma_{2}=0}^{N}\left(\begin{array}{c}
N \\
\sigma_{2}
\end{array}\right)\left(\frac{t}{n}\right)^{\sigma_{2}}\left(1-\frac{t}{n}\right)^{N-\sigma_{2}},
\end{array}\right.
$$

where $N=2^{\psi} n$.

Hence the Bernstein polynomial patch for the points $\left\{\varphi_{\sigma_{1}, \sigma_{2}}^{\psi}\right\}$ on $[0, n] \times[0, n]$ is

$$
b_{\psi}(r, t)=\left[b_{\psi_{1}}(r) \times b_{\psi_{2}}(t)\right] \varphi_{\sigma_{1}, \sigma_{2}}^{\psi} .
$$


If we take derivatives of the first and second equations in (17) with respect to $r$ and $t$, respectively, we obtain the following two equations:

$$
\begin{aligned}
b_{\psi_{1}}^{\prime}(r)= & \sum_{\sigma_{1}=0}^{N}\left(\begin{array}{l}
N \\
\sigma_{1}
\end{array}\right) \sigma_{1}\left(\frac{1}{n}\right)\left(\frac{r}{n}\right)^{\sigma_{1}-1}\left(1-\frac{r}{n}\right)^{N-\sigma_{1}} \\
& +\sum_{\sigma_{1}=0}^{N}\left(\begin{array}{l}
N \\
\sigma_{1}
\end{array}\right)\left(\frac{r}{n}\right)^{\sigma_{1}}\left(N-\sigma_{1}\right)\left(1-\frac{r}{n}\right)^{N-\sigma_{1}-1}\left(-\frac{1}{n}\right), \\
b_{\psi_{2}}^{\prime}(t)= & \sum_{\sigma_{2}=0}^{N}\left(\begin{array}{l}
N \\
\sigma_{2}
\end{array}\right) \sigma_{2}\left(\frac{1}{n}\right)\left(\frac{t}{n}\right)^{\sigma_{2}-1}\left(1-\frac{t}{n}\right)^{N-\sigma_{2}} \\
& +\sum_{\sigma_{2}=0}^{N}\left(\begin{array}{l}
N \\
\sigma_{2}
\end{array}\right)\left(\frac{t}{n}\right)^{\sigma_{2}}\left(N-\sigma_{2}\right)\left(1-\frac{t}{n}\right)^{N-\sigma_{2}-1}\left(-\frac{1}{n}\right) .
\end{aligned}
$$

Now we calculate $B_{\psi}(r, t)$ which is the mesh with basis functions $b_{\psi_{1}}^{\prime}(r) \times b_{\psi_{2}}^{\prime}(t)$,

$$
B_{\psi}(r, t)=\left[b_{\psi_{1}}^{\prime}(r) \times b_{\psi_{2}}^{\prime}(t)\right] \varphi_{\sigma_{1}, \sigma_{2}}^{\psi} .
$$

Thus firstly we use (19) and (20) in (21), and then apply some simple algebraic operations and obtain

$$
B_{\psi}(r, t)=\overline{B_{1}}+\bar{B}_{2}+\bar{B}_{3}+\bar{B}_{4},
$$

where

$$
\begin{aligned}
\overline{B_{1}}= & \frac{N^{2}}{n^{2}} \sum_{\sigma_{1}=0}^{N-1} \frac{(N-1) !}{\sigma_{1} !\left(N-\sigma_{1}-1\right) !}\left(\frac{r}{n}\right)^{\sigma_{1}}\left(1-\frac{r}{n}\right)^{N-\sigma_{1}-1} \\
& \times \sum_{\sigma_{2}=0}^{N-1} \frac{(N-1) !}{\sigma_{2} !\left(N-\sigma_{2}-1\right) !}\left(\frac{t}{n}\right)^{\sigma_{2}}\left(1-\frac{t}{n}\right)^{N-\sigma_{2}-1} \varphi_{\sigma_{1}+1, \sigma_{2}+1}^{\psi}, \\
\overline{B_{2}}= & -\frac{N^{2}}{n^{2}} \sum_{\sigma_{1}=0}^{N-1} \frac{N !}{\sigma_{1} !\left(N-\sigma_{1}-1\right) !}\left(\frac{r}{n}\right)^{\sigma_{1}}\left(1-\frac{r}{n}\right)^{N-\sigma_{1}-1} \\
& \times \sum_{\sigma_{2}=0}^{N-1} \frac{(N-1) !}{\sigma_{2} !\left(N-\sigma_{2}-1\right) !}\left(\frac{t}{n}\right)^{\sigma_{2}}\left(1-\frac{t}{n}\right)^{N-\sigma_{2}-1} \varphi_{\sigma_{1}, \sigma_{2}+1}^{\psi}, \\
\overline{B_{3}=} & -\frac{N^{2}}{n^{2}} \sum_{\sigma_{1}=0}^{N-1} \frac{(N-1) !}{\sigma_{1} !\left(N-\sigma_{1}-1\right) !}\left(\frac{r}{n}\right)^{\sigma_{1}}\left(1-\frac{r}{n}\right)^{N-\sigma_{1}-1} \\
& \times \sum_{\sigma_{2}=0}^{N-1} \frac{N !}{\sigma_{2} !\left(N-\sigma_{2}-1\right) !}\left(\frac{t}{n}\right)^{\sigma_{2}}\left(1-\frac{t}{n}\right)^{N-\sigma_{2}-1} \varphi_{\sigma_{1}+1, \sigma_{2}}^{\psi}, \\
\overline{B_{4}=}= & N^{2} \sum_{n^{2}}^{N-1} \frac{N !}{\sigma_{1}=0}\left(\frac{r}{\sigma_{1} !\left(N-\sigma_{1}-1\right) !}\left(\frac{r}{n}\right)^{\sigma_{1}}\left(1-\frac{r}{n}\right)^{N-\sigma_{1}-1}\right. \\
& \times \sum_{\sigma_{2}=0}^{N-1} \frac{N !}{\sigma_{2} !\left(N-\sigma_{2}-1\right) !}\left(\frac{t}{n}\right)^{\sigma_{2}}\left(1-\frac{t}{n}\right)^{N-\sigma_{2}-1} \varphi_{\sigma_{1}, \sigma_{2}}^{\psi} .
\end{aligned}
$$


This gives us

$$
\begin{aligned}
B_{\psi}(r, t)= & \frac{N^{2}}{n^{2}} \sum_{\sigma_{1}=0}^{N-1}\left(\begin{array}{c}
N-1 \\
\sigma_{1}
\end{array}\right)\left(\frac{r}{n}\right)^{\sigma_{1}}\left(1-\frac{r}{n}\right)^{N-\sigma_{1}-1} \sum_{\sigma_{2}=0}^{N-1}\left(\begin{array}{c}
N-1 \\
\sigma_{2}
\end{array}\right) \\
& \times\left(\frac{t}{n}\right)^{\sigma_{2}}\left(1-\frac{t}{n}\right)^{N-\sigma_{2}-1}\left(\varphi_{\sigma_{1}+1, \sigma_{2}+1}^{\psi}-\varphi_{\sigma_{1}, \sigma_{2}+1}^{\psi}-\varphi_{\sigma_{1}+1, \sigma_{2}}^{\psi}+\varphi_{\sigma_{1}, \sigma_{2}}^{\psi}\right) .
\end{aligned}
$$

Since $N^{2}=\left(2^{\psi}\right)^{2} n^{2}$, we get

$$
\begin{aligned}
B_{\psi}(r, t)= & \sum_{\sigma_{1}=0}^{N-1}\left(\begin{array}{c}
N-1 \\
\sigma_{1}
\end{array}\right)\left(\frac{r}{n}\right)^{\sigma_{1}}\left(1-\frac{r}{n}\right)^{N-\sigma_{1}-1} \sum_{\sigma_{2}=0}^{N-1}\left(\begin{array}{c}
N-1 \\
\sigma_{2}
\end{array}\right) \\
& \times\left(\frac{t}{n}\right)^{\sigma_{2}}\left(1-\frac{t}{n}\right)^{N-\sigma_{2}-1} E_{\sigma_{1}, \sigma_{2}}^{\psi},
\end{aligned}
$$

where

$$
E_{\sigma_{1}, \sigma_{2}}^{\psi}=2^{2 \psi}(1 !)^{-2} \Delta_{\sigma_{1}} \Delta_{\sigma_{2}} \varphi_{\sigma_{1}, \sigma_{2}}^{\psi} .
$$

The Bernstein polynomial batches are uniformly convergent, therefore $\lim _{\psi \rightarrow \infty} B_{\psi}=\varphi$ and $\lim _{\psi \rightarrow \infty} B_{\psi}^{\prime}=E$ on $[0, n] \times[0, n]$. Hence $\varphi^{\prime}=E \in \Gamma_{[0, n]^{2}}$. This completes our proof.

Similarly, the following lemma can be proved.

Lemma 6 Let $\varphi^{\psi}=\left\{\left(\frac{\sigma_{1}}{2 \psi}, \frac{\sigma_{2}}{2 \psi}, \varphi_{\sigma_{1}, \sigma_{2}}^{\psi}\right): \sigma_{1}, \sigma_{2} \in \mathbb{Z}, \psi \in \mathbb{N}\right\}$ be a mesh produced by the GTPBRS at the $\psi$ th refinement level and $H^{\psi}=\left\{\left(\frac{\sigma_{1}}{2^{\psi}}, \frac{\sigma_{2}}{2^{\psi}}, H_{\sigma_{1}, \sigma_{2}}^{\psi}\right): \sigma_{1}, \sigma_{2} \in \mathbb{Z}, \psi \in \mathbb{N}\right\}$ be a mesh produced by the second order GBDDRS at the $\psi$ th refinement level. If $\lim _{\psi \rightarrow \infty} \varphi^{\psi}=\varphi \in \Gamma_{[0, n]^{2}}$, and $\varphi$ is the limiting model produced by GTPBRS then $H=\varphi^{\prime \prime}$.

\section{The deviation of tensor product binary and its DD refinement schemes}

In this section, first we introduce the inequalities to compute the deviation between two consecutive points at the same refinement level. Then we introduce the inequalities to compute the deviation between successive levels of meshes produced by GTPBRS and its GDDBRSs.

\subsection{The deviation of GTPBRS}

In this subsection, we first introduce the inequalities to compute the deviation between two consecutive points at the $(\psi+1)$ th refinement level then we introduce the inequalities to compute the deviation between the $\psi$ th and $(\psi+1)$ th level of meshes produced by GTPBRS.

Lemma 7 Let $\varphi^{0}=\left\{\left(\sigma_{1}, \sigma_{2}, \varphi_{\sigma_{1}, \sigma_{2}}^{0}\right): \sigma_{1}, \sigma_{2} \in \mathbb{Z}\right\}$ be an initial mesh and $\varphi^{\psi+1}=\left\{\left(\frac{\sigma_{1}}{2^{\psi+1}}, \frac{\sigma_{2}}{2^{\psi+1}}\right.\right.$, $\left.\left.\varphi_{\sigma_{1}, \sigma_{2}}^{\psi+1}\right): \sigma_{1}, \sigma_{2} \in \mathbb{Z}, \psi \in \mathbb{N}\right\}$ be a mesh produced by the GTPBRS at the $(\psi+1)$ th refinement level. If we fix $m=3$, then the deviations between two consecutive points at the $(\psi+1)$ th refinement step are

$$
\left\{\begin{array}{l}
\max _{\sigma_{1}, \sigma_{2}}\left\|\varphi_{\sigma_{1}+1, \sigma_{2}}^{\psi+1}-\varphi_{\sigma_{1}, \sigma_{2}}^{\psi+1}\right\| \leq(T)^{\psi+1} \max _{\sigma_{1}, \sigma_{2}}\left\|\varphi_{\sigma_{1}+1, \sigma_{2}}^{0}-\varphi_{\sigma_{1}, \sigma_{2}}^{0}\right\|, \\
\max _{\sigma_{1}, \sigma_{2}}\left\|\varphi_{\sigma_{1}, \sigma_{2}+1}^{\psi+1}-\varphi_{\sigma_{1}, \sigma_{2}}^{\psi+1}\right\| \leq(T)^{\psi+1} \max _{\sigma_{1}, \sigma_{2}}\left\|\varphi_{\sigma_{1}, \sigma_{2}+1}^{0}-\varphi_{\sigma_{1}, \sigma_{2}}^{0}\right\|
\end{array}\right.
$$


where

$$
T=\max _{\theta}\left\{T_{\theta}: \theta=1,2,3,4\right\}
$$

and

$$
\left\{\begin{array}{l}
T_{1}=\left|\sum_{l=0}^{3} \rho_{l}\right| \sum_{t=0}^{2}\left|\sum_{u=0}^{t}\left(\rho_{t-u}-\chi_{t-u}\right)\right|, \\
T_{2}=\left|\sum_{l=0}^{3} \rho_{l}\right| \sum_{t=0}^{3}\left|\sum_{u=0}^{t}\left(\chi_{t-u}-\rho_{t-u-1}\right)\right|, \\
T_{3}=\left|\sum_{l=0}^{3} \chi_{l}\right| \sum_{t=0}^{2}\left|\sum_{u=0}^{t}\left(\rho_{t-u}-\chi_{t-u}\right)\right|, \\
T_{4}=\left|\sum_{l=0}^{3} \chi_{l}\right| \sum_{t=0}^{3}\left|\sum_{u=0}^{t}\left(\chi_{t-u}-\rho_{t-u-1}\right)\right| .
\end{array}\right.
$$

Proof To prove this lemma, we have to calculate distances between every two consecutive points in $\sigma_{1}$ direction and similarly in $\sigma_{2}$ direction. So by Fig. 1 , we have to calculate 12 differences to prove the required result.

First, we calculate the difference between second and first refinement rules of the GTPBRS defined in (1) for a fixed $m=3$, thus we get

$$
\begin{aligned}
\varphi_{2 \sigma_{1}+1,2 \sigma_{2}}^{\psi+1}-\varphi_{2 \sigma_{1}, 2 \sigma_{2}}^{\psi+1} & =\sum_{r=0}^{3} \sum_{s=0}^{3} \chi_{r} \rho_{s} \varphi_{\sigma_{1}+r, \sigma_{2}+s}^{\psi}-\sum_{r=0}^{3} \sum_{s=0}^{3} \rho_{r} \rho_{s} \varphi_{\sigma_{1}+r, \sigma_{2}+s}^{\psi} \\
& =\sum_{r=0}^{3} \sum_{s=0}^{3}\left(\chi_{r}-\rho_{r}\right) \rho_{s} \varphi_{\sigma_{1}+r, \sigma_{2}+s^{*}}^{\psi}
\end{aligned}
$$

By expanding the above expression, we get

$$
\begin{aligned}
\varphi_{2 \sigma_{1}+1,2 \sigma_{2}}^{\psi+1}-\varphi_{2 \sigma_{1}, 2 \sigma_{2}}^{\psi+1}= & \rho_{0}\left\{\omega_{0}\left(\varphi_{\sigma_{1}+1, \sigma_{2}}^{\psi}-\varphi_{\sigma_{1}, \sigma_{2}}^{\psi}\right)+\omega_{1}\left(\varphi_{\sigma_{1}+2, \sigma_{2}}^{\psi}-\varphi_{\sigma_{1}+1, \sigma_{2}}^{\psi}\right)+\omega_{2}\left(\varphi_{\sigma_{1}+3, \sigma_{2}}^{\psi}\right.\right. \\
& \left.\left.-\varphi_{\sigma_{1}+2, \sigma_{2}}^{\psi}\right)+\omega_{3} \varphi_{\sigma_{1}+3, \sigma_{2}}^{\psi}\right\}+\rho_{1}\left\{\omega_{0}\left(\varphi_{\sigma_{1}+1, \sigma_{2}+1}^{\psi}-\varphi_{\sigma_{1}, \sigma_{2}+1}^{\psi}\right)+\omega_{1}\right. \\
& \times\left(\varphi_{\sigma_{1}+2, \sigma_{2}+1}^{\psi}-\varphi_{\sigma_{1}+1, \sigma_{2}+1}^{\psi}\right)+\omega_{2}\left(\varphi_{\sigma_{1}+3, \sigma_{2}+1}^{\psi}-\varphi_{\sigma_{1}+2, \sigma_{2}+1}^{\psi}\right)+\omega_{3} \\
& \left.\times \varphi_{\sigma_{1}+3, \sigma_{2}+1}^{\psi}\right\}+\rho_{2}\left\{\omega_{0}\left(\varphi_{\sigma_{1}+1, \sigma_{2}+2}^{\psi}-\varphi_{\sigma_{1}, \sigma_{2}+2}^{\psi}\right)+\omega_{1}\left(\varphi_{\sigma_{1}+2, \sigma_{2}+2}^{\psi}\right.\right. \\
& \left.\left.-\varphi_{\sigma_{1}+1, \sigma_{2}+2}^{\psi}\right)+\omega_{2}\left(\varphi_{\sigma_{1}+3, \sigma_{2}+2}^{\psi}-\varphi_{\sigma_{1}+2, \sigma_{2}+2}^{\psi}\right)+\omega_{3} \varphi_{\sigma_{1}+3, \sigma_{2}+2}^{\psi}\right\}+\rho_{3} \\
& \times\left\{\omega_{0}\left(\varphi_{\sigma_{1}+1, \sigma_{2}+3}^{\psi}-\varphi_{\sigma_{1}, \sigma_{2}+3}^{\psi}\right)+\omega_{1}\left(\varphi_{\sigma_{1}+2, \sigma_{2}+3}^{\psi}-\varphi_{\sigma_{1}+1, \sigma_{2}+3}^{\psi}\right)+\omega_{2}\right. \\
& \left.\times\left(\varphi_{\sigma_{1}+3, \sigma_{2}+3}^{\psi}-\varphi_{\sigma_{1}+2, \sigma_{2}+3}^{\psi}\right)+\omega_{3} \varphi_{\sigma_{1}+3, \sigma_{2}+3}^{\psi}\right\},
\end{aligned}
$$

where

$$
\begin{cases}\omega_{0}=\left(\sum_{l=0}^{0} \rho_{l}-\sum_{l=0}^{0} \chi_{l}\right), & \omega_{1}=\left(\sum_{l=0}^{1} \rho_{l}-\sum_{l=0}^{1} \chi_{l}\right) \\ \omega_{2}=\left(\sum_{l=0}^{2} \rho_{l}-\sum_{l=0}^{2} \chi_{l}\right), & \omega_{3}=\left(\sum_{l=0}^{3} \rho_{l}-\sum_{l=0}^{3} \chi_{l}\right)\end{cases}
$$

Since $\omega_{3}=0$ by (2), we get

$$
\varphi_{2 \sigma_{1}+1,2 \sigma_{2}}^{\psi+1}-\varphi_{2 \sigma_{1}, 2 \sigma_{2}}^{\psi+1}=\sum_{l=0}^{3}\left[\rho_{l} \sum_{t=0}^{2}\left\{\sum_{u=0}^{t}\left(\rho_{t-u}-\chi_{t-u}\right)\left(\varphi_{\sigma_{1}+t+1, \sigma_{2}+l}^{\psi}-\varphi_{\sigma_{1}+t, \sigma_{2}+l}^{\psi}\right)\right\}\right] .
$$


Now by calculating the infinity norm, we get

$$
\left\|\varphi_{2 \sigma_{1}+1,2 \sigma_{2}}^{\psi+1}-\varphi_{2 \sigma_{1}, 2 \sigma_{2}}^{\psi+1}\right\| \leq T_{1} \max _{\sigma_{1}, \sigma_{2}}\left\|\varphi_{\sigma_{1}+1, \sigma_{2}}^{\psi}-\varphi_{\sigma_{1}, \sigma_{2}}^{\psi}\right\|
$$

where

$$
T_{1}=\left|\sum_{l=0}^{3} \rho_{l}\right| \sum_{t=0}^{2}\left|\sum_{u=0}^{t}\left(\rho_{t-u}-\chi_{t-u}\right)\right| .
$$

Similarly, by using (1) for $m=3$ and adopting similar procedures to above, we get the following 11 inequalities:

$$
\left\|\varphi_{2 \sigma_{1}+2,2 \sigma_{2}}^{\psi+1}-\varphi_{2 \sigma_{1}+1,2 \sigma_{2}}^{\psi+1}\right\| \leq T_{2} \max _{\sigma_{1}, \sigma_{2}}\left\|\varphi_{\sigma_{1}+1, \sigma_{2}}^{\psi}-\varphi_{\sigma_{1}, \sigma_{2}}^{\psi}\right\|
$$

where

$$
\begin{aligned}
& T_{2}=\left|\sum_{l=0}^{3} \rho_{l}\right| \sum_{t=0}^{3}\left|\sum_{u=0}^{t}\left(\chi_{t-u}-\rho_{t-u-1}\right)\right|, \\
& \left\|\varphi_{2 \sigma_{1}+1,2 \sigma_{2}+1}^{\psi+1}-\varphi_{2 \sigma_{1}, 2 \sigma_{2}+1}^{\psi+1}\right\| \leq T_{3} \max _{\sigma_{1}, \sigma_{2}}\left\|\varphi_{\sigma_{1}+1, \sigma_{2}}^{\psi}-\varphi_{\sigma_{1}, \sigma_{2}}^{\psi}\right\|,
\end{aligned}
$$

where

$$
\begin{aligned}
& T_{3}=\left|\sum_{l=0}^{3} \chi_{l}\right| \sum_{t=0}^{2}\left|\sum_{u=0}^{t}\left(\rho_{t-u}-\chi_{t-u}\right)\right|, \\
& \left\|\varphi_{2 \sigma_{1}+2,2 \sigma_{2}+1}^{\psi+1}-\varphi_{2 \sigma_{1}+1,2 \sigma_{2}+1}^{\psi+1}\right\| \leq T_{4} \max _{\sigma_{1}, \sigma_{2}}\left\|\varphi_{\sigma_{1}+1, \sigma_{2}}^{\psi}-\varphi_{\sigma_{1}, \sigma_{2}}^{\psi}\right\|,
\end{aligned}
$$

where

$$
\begin{aligned}
& T_{4}=\left|\sum_{l=0}^{3} \chi_{l}\right| \sum_{t=0}^{3}\left|\sum_{u=0}^{t}\left(\chi_{t-u}-\rho_{t-u-1}\right)\right|, \\
& \left\|\varphi_{2 \sigma_{1}+1,2 \sigma_{2}+2}^{\psi+1}-\varphi_{2 \sigma_{1}, 2 \sigma_{2}+2}^{\psi+1}\right\| \leq T_{1} \max _{\sigma_{1}, \sigma_{2}}\left\|\varphi_{\sigma_{1}+1, \sigma_{2}+1}^{\psi}-\varphi_{\sigma_{1}, \sigma_{2}+1}^{\psi}\right\|,
\end{aligned}
$$

where

$$
\begin{aligned}
& T_{1}=\left|\sum_{l=0}^{3} \rho_{l}\right| \sum_{t=0}^{2}\left|\sum_{u=0}^{t}\left(\rho_{t-u}-\chi_{t-u}\right)\right|, \\
& \left\|\varphi_{2 \sigma_{1}+2,2 \sigma_{2}+2}^{\psi+1}-\varphi_{2 \sigma_{1}+1,2 \sigma_{2}+2}^{\psi+1}\right\| \leq T_{2} \max _{\sigma_{1}, \sigma_{2}}\left\|\varphi_{\sigma_{1}+1, \sigma_{2}+1}^{\psi}-\varphi_{\sigma_{1}, \sigma_{2}+1}^{\psi}\right\|,
\end{aligned}
$$

where

$$
\begin{aligned}
& T_{2}=\left|\sum_{l=0}^{3} \rho_{l}\right| \sum_{t=0}^{3}\left|\sum_{u=0}^{t}\left(\chi_{t-u}-\rho_{t-u-1}\right)\right|, \\
& \left\|\varphi_{2 \sigma_{1}, 2 \sigma_{2}+1}^{\psi+1}-\varphi_{2 \sigma_{1}, 2 \sigma_{2}}^{\psi+1}\right\| \leq T_{1} \max _{\sigma_{1}, \sigma_{2}}\left\|\varphi_{\sigma_{1}, \sigma_{2}+l}^{\psi}-\varphi_{\sigma_{1}, \sigma_{2}}^{\psi}\right\|,
\end{aligned}
$$


where

$$
\begin{aligned}
& T_{1}=\left|\sum_{l=0}^{3} \rho_{l}\right| \sum_{t=0}^{2}\left|\sum_{u=0}^{t}\left(\rho_{t-u}-\chi_{t-u}\right)\right|, \\
& \left\|\varphi_{2 \sigma_{1}, 2 \sigma_{2}+2}^{\psi+1}-\varphi_{2 \sigma_{1}, 2 \sigma_{2}+1}^{\psi+1}\right\| \leq T_{2} \max _{\sigma_{1}, \sigma_{2}}\left\|\varphi_{\sigma_{1}, \sigma_{2}+l}^{\psi}-\varphi_{\sigma_{1}, \sigma_{2}}^{\psi}\right\|,
\end{aligned}
$$

where

$$
\begin{aligned}
& T_{2}=\left|\sum_{l=0}^{3} \rho_{l}\right| \sum_{t=0}^{3}\left|\sum_{u=0}^{t}\left(\chi_{t-u}-\rho_{t-u-1}\right)\right|, \\
& \left\|\varphi_{2 \sigma_{1}+1,2 \sigma_{2}+1}^{\psi+1}-\varphi_{2 \sigma_{1}+1,2 \sigma_{2}}^{\psi+1}\right\| \leq T_{3} \max _{\sigma_{1}, \sigma_{2}}\left\|\varphi_{\sigma_{1}, \sigma_{2}+l}^{\psi}-\varphi_{\sigma_{1}, \sigma_{2}}^{\psi}\right\|,
\end{aligned}
$$

where

$$
\begin{aligned}
& T_{3}=\left|\sum_{l=0}^{3} \chi_{l}\right| \sum_{t=0}^{2}\left|\sum_{u=0}^{t}\left(\rho_{t-u}-\chi_{t-u}\right)\right|, \\
& \left\|\varphi_{2 \sigma_{1}+1,2 \sigma_{2}+2}^{\psi+1}-\varphi_{2 \sigma_{1}+1,2 \sigma_{2}+1}^{\psi+1}\right\| \leq T_{4} \max _{\sigma_{1}, \sigma_{2}}\left\|\varphi_{\sigma_{1}, \sigma_{2}+1}^{\psi}-\varphi_{\sigma_{1}, \sigma_{2}}^{\psi}\right\|,
\end{aligned}
$$

where

$$
\begin{aligned}
& T_{4}=\left|\sum_{l=0}^{3} \chi_{l}\right| \sum_{t=0}^{3}\left|\sum_{u=0}^{t}\left(\chi_{t-u}-\rho_{t-u-1}\right)\right|, \\
& \left\|\varphi_{2 \sigma_{1}+2,2 \sigma_{2}+1}^{\psi+1}-\varphi_{2 \sigma_{1}+2,2 \sigma_{2}}^{\psi+1}\right\| \leq T_{1} \max _{\sigma_{1}, \sigma_{2}}\left\|\varphi_{\sigma_{1}, \sigma_{2}+l}^{\psi}-\varphi_{\sigma_{1}, \sigma_{2}}^{\psi}\right\|,
\end{aligned}
$$

where

$$
\begin{aligned}
& T_{1}=\left|\sum_{l=0}^{3} \rho_{l}\right| \sum_{t=0}^{2}\left|\sum_{u=0}^{t}\left(\rho_{t-u}-\chi_{t-u}\right)\right|, \\
& \left\|\varphi_{2 \sigma_{1}+2,2 \sigma_{2}+2}^{\psi+1}-\varphi_{2 \sigma_{1}+2,2 \sigma_{2}+1}^{\psi+1}\right\| \leq T_{2} \max _{\sigma_{1}, \sigma_{2}}\left\|\varphi_{\sigma_{1}, \sigma_{2}+l}^{\psi}-\varphi_{\sigma_{1}, \sigma_{2}}^{\psi}\right\|,
\end{aligned}
$$

where

$$
T_{2}=\left|\sum_{l=0}^{3} \rho_{l}\right| \sum_{t=0}^{3}\left|\sum_{u=0}^{t}\left(\chi_{t-u}-\rho_{t-u-1}\right)\right|
$$

Now by combining (26)-(37), we get

$$
\begin{aligned}
& \max _{\sigma_{1}, \sigma_{2}}\left\|\varphi_{\sigma_{1}+1, \sigma_{2}}^{\psi+1}-\varphi_{\sigma_{1}, \sigma_{2}}^{\psi+1}\right\| \leq T \max _{\sigma_{1}, \sigma_{2}}\left\|\varphi_{\sigma_{1}+1, \sigma_{2}}^{\psi}-\varphi_{\sigma_{1}, \sigma_{2}}^{\psi}\right\|, \\
& \max _{\sigma_{1}, \sigma_{2}}^{\psi}\left\|\varphi_{\sigma_{1}, \sigma_{2}+1}^{\psi+1}-\varphi_{\sigma_{1}, \sigma_{2}}^{\psi+1}\right\| \leq T \max _{\sigma_{1}, \sigma_{2}}\left\|\varphi_{\sigma_{1}, \sigma_{2}+1}^{\psi}-\varphi_{\sigma_{1}, \sigma_{2}}^{\psi}\right\|,
\end{aligned}
$$

where

$$
T=\max \left\{T_{1}, T_{2}, T_{3} \cdot T_{4}\right\} .
$$


This further implies that

$$
\begin{aligned}
& \max _{\sigma_{1}, \sigma_{2}}\left\|\varphi_{\sigma_{1}+1, \sigma_{2}}^{\psi+1}-\varphi_{\sigma_{1}, \sigma_{2}}^{\psi+1}\right\| \leq(T)^{\psi+1} \max _{\sigma_{1}, \sigma_{2}}\left\|\varphi_{\sigma_{1}+1, \sigma_{2}}^{0}-\varphi_{\sigma_{1}, \sigma_{2}}^{0}\right\|, \\
& \max _{\sigma_{1}, \sigma_{2}}\left\|\varphi_{\sigma_{1}, \sigma_{2}+1}^{\psi+1}-\varphi_{\sigma_{1}, \sigma_{2}}^{\psi+1}\right\| \leq(T)^{\psi+1} \max _{\sigma_{1}, \sigma_{2}}\left\|\varphi_{\sigma_{1}, \sigma_{2}+1}^{0}-\varphi_{\sigma_{1}, \sigma_{2}}^{0}\right\| .
\end{aligned}
$$

This completes the proof.

Lemma 8 Let $\varphi^{\psi}=\left\{\left(\frac{\sigma_{1}}{2^{\psi}}, \frac{\sigma_{2}}{2^{\psi}}, \varphi_{\sigma_{1}, \sigma_{2}}^{\psi}\right): \sigma_{1}, \sigma_{2} \in \mathbb{Z}, \psi \in \mathbb{N}\right\}$ be a mesh at the $\psi$ th refinement step and $\varphi^{\psi+1}=\left\{\left(\frac{\sigma_{1}}{2^{\psi+1}}, \frac{\sigma_{2}}{2^{\psi+1}}, \varphi_{\sigma_{1}, \sigma_{2}}^{\psi+1}\right): \sigma_{1}, \sigma_{2} \in \mathbb{Z}, \psi \in \mathbb{N}\right\}$ be a mesh produced by the GTPBRS at the $(\psi+1)$ th refinement level. If we fix $m=3$, then the deviation between two consecutive meshes at the $\psi$ th and $(\psi+1)$ th refinement steps is

$$
\left\|\varphi^{\psi+1}-\varphi^{\psi}\right\|_{\infty} \leq \delta\left\{\max _{\sigma_{1}, \sigma_{2}}\left\|\varphi_{\sigma_{1}+1, \sigma_{2}}^{\psi}-\varphi_{\sigma_{1}, \sigma_{2}}^{\psi}\right\|+\max _{\sigma_{1}, \sigma_{2}}\left\|\varphi_{\sigma_{1}, \sigma_{2}+1}^{\psi}-\varphi_{\sigma_{1}, \sigma_{2}}^{\psi}\right\|\right\},
$$

where

$$
\begin{aligned}
& \delta=\max _{\theta}\left\{\delta_{\theta}: \theta=1,2,3,4\right\}, \\
& \left\{\begin{array}{l}
\delta_{1}=\left|3-\left(\rho_{2}+2 \rho_{1}+3 \rho_{0}\right)\right|, \\
\delta_{2}=\left|3-\left(\rho_{2}+2 \rho_{1}+3 \rho_{0}\right)\right|, \\
\delta_{3}=\left|\frac{5}{2}-\left(\chi_{2}+2 \chi_{1}+3 \chi_{0}\right)\right|, \\
\delta_{4}=\left|\frac{5}{2}-\left(\chi_{2}+2 \chi_{1}+3 \chi_{0}\right)\right| .
\end{array}\right.
\end{aligned}
$$

Proof To find the deviation between two consecutive meshes at the $\psi$ th and $(\psi+1)$ th refinement steps, we have to calculate

$$
\left\|\varphi^{\psi+1}-\varphi^{\psi}\right\|_{\infty} \leq \max \left\{N_{1}^{\psi}, N_{2}^{\psi}, N_{3}^{\psi}, N_{4}^{\psi}\right\}
$$

where

$$
\left\{\begin{array}{l}
N_{1}^{\psi}=\max _{\sigma_{1}, \sigma_{2}}\left\|\varphi_{2 \sigma_{1}, 2 \sigma_{2}}^{\psi+1}-\varphi_{\sigma_{1}, \sigma_{2}}^{\psi}\right\|, \\
N_{2}^{\psi}=\max _{\sigma_{1}, \sigma_{2}}\left\|\varphi_{2 \sigma_{1}+1,2 \sigma_{2}}^{\psi+1}-\frac{1}{2}\left(\varphi_{\sigma_{1}, \sigma_{2}}^{\psi}+\varphi_{\sigma_{1}+1, \sigma_{2}}^{\psi}\right)\right\|, \\
N_{3}^{\psi}=\max _{\sigma_{1}, \sigma_{2}}\left\|\varphi_{2 \sigma_{1}, 2 \sigma_{2}+1}^{\psi+1}-\frac{1}{2}\left(\varphi_{\sigma_{1}, \sigma_{2}}^{\psi}+\varphi_{\sigma_{1}, \sigma_{2}+1}^{\psi}\right)\right\|, \\
N_{4}^{\psi}=\max _{\sigma_{1}, \sigma_{2}}\left\|\varphi_{2 \sigma_{1}+1,2 \sigma_{2}+1}^{\psi+1}-\frac{1}{4}\left(\varphi_{\sigma_{1}, \sigma_{2}}^{\psi}+\varphi_{\sigma_{1}+1, \sigma_{2}}^{\psi}+\varphi_{\sigma_{1}, \sigma_{2}+1}^{\psi}+\varphi_{\sigma_{1}+1, \sigma_{2}+1}^{\psi}\right)\right\| .
\end{array}\right.
$$

By using the refinement rule of (1) for a fixed $m=3$, we obtain

$$
\varphi_{2 \sigma_{1}, 2 \sigma_{2}}^{\psi+1}-\varphi_{\sigma_{1}, \sigma_{2}}^{\psi}=\sum_{r=0}^{3} \sum_{s=0}^{3} \rho_{r} \rho_{s} \varphi_{\sigma_{1}+r, \sigma_{2}+s}^{\psi}-\varphi_{\sigma_{1}, \sigma_{2}}^{\psi} .
$$

By expanding the above expression, we get

$$
\varphi_{2 \sigma_{1}, 2 \sigma_{2}}^{\psi+1}-\varphi_{\sigma_{1}, \sigma_{2}}^{\psi}=\bar{M}_{1}+\bar{M}_{2}+\bar{M}_{3}
$$


where

$$
\begin{aligned}
\bar{M}_{1}= & \left(1-\rho_{0} \rho_{0}\right)\left(\varphi_{\sigma_{1}+1, \sigma_{2}}^{\psi}-\varphi_{\sigma_{1}, \sigma_{2}}^{\psi}\right)+\left(1-\rho_{0} \sum_{l=0}^{1} \rho_{l}\right)\left(\varphi_{\sigma_{1}+2, \sigma_{2}}^{\psi}-\varphi_{\sigma_{1}+1, \sigma_{2}}^{\psi}\right) \\
& +\left(1-\rho_{0} \sum_{l=0}^{2} \rho_{l}\right)\left(\varphi_{\sigma_{1}+3, \sigma_{2}}^{\psi}-\varphi_{\sigma_{1}+2, \sigma_{2}}^{\psi}\right)+\rho_{1}\left\{-\rho_{0}\left(\varphi_{\sigma_{1}+1, \sigma_{2}+1}^{\psi}-\varphi_{\sigma_{1}, \sigma_{2}+1}^{\psi}\right)\right. \\
& \left.-\sum_{l=0}^{1} \rho_{l}\left(\varphi_{\sigma_{1}+2, \sigma_{2}+1}^{\psi}-\varphi_{\sigma_{1}+1, \sigma_{2}+1}^{\psi}\right)-\sum_{l=0}^{2} \rho_{l}\left(\varphi_{\sigma_{1}+3, \sigma_{2}+1}^{\psi}-\varphi_{\sigma_{1}+2, \sigma_{2}+1}^{\psi}\right)\right\}, \\
\bar{M}_{2}= & \rho_{2}\left\{-\rho_{0}\left(\varphi_{\sigma_{1}+1, \sigma_{2}+2}^{\psi}-\varphi_{\sigma_{1}, \sigma_{2}+2}^{\psi}\right)-\sum_{l=0}^{1} \rho_{l}\left(\varphi_{\sigma_{1}+2, \sigma_{2}+2}^{\psi}-\varphi_{\sigma_{1}+1, \sigma_{2}+2}^{\psi}\right)\right. \\
& \left.-\sum_{l=0}^{2} \rho_{l}\left(\varphi_{\sigma_{1}+3, \sigma_{2}+2}^{\psi}-\varphi_{\sigma_{1}+2, \sigma_{2}+2}^{\psi}\right)\right\}+\rho_{3}\left\{-\rho_{0}\left(\varphi_{\sigma_{1}+1, \sigma_{2}+3}^{\psi}-\varphi_{\sigma_{1}, \sigma_{2}+3}^{\psi}\right)\right. \\
& \left.-\sum_{l=0}^{1} \rho_{l}\left(\varphi_{\sigma_{1}+2, \sigma_{2}+3}^{\psi}-\varphi_{\sigma_{1}+1, \sigma_{2}+3}^{\psi}\right)-\sum_{l=0}^{2} \rho_{l}\left(\varphi_{\sigma_{1}+3, \sigma_{2}+3}^{\psi}-\varphi_{\sigma_{1}+2, \sigma_{2}+3}^{\psi}\right)\right\}
\end{aligned}
$$

and

$$
\begin{aligned}
\bar{M}_{3}= & \left(1-\rho_{0} \sum_{l=0}^{3} \rho_{l}\right)\left(\varphi_{\sigma_{1}+3, \sigma_{2}+1}^{\psi}-\varphi_{\sigma_{1}+3, \sigma_{2}}^{\psi}\right)+\left(1-\sum_{l=0}^{1} \rho_{l} \sum_{l=0}^{3} \rho_{l}\right) \\
& \times\left(\varphi_{\sigma_{1}+3, \sigma_{2}+2}^{\psi}-\varphi_{\sigma_{1}+3, \sigma_{2}+1}^{\psi}\right)+\left(1-\sum_{l=0}^{2} \rho_{l} \sum_{l=0}^{3} \rho_{l}\right)\left(\varphi_{\sigma_{1}+3, \sigma_{2}+2}^{\psi}-\varphi_{\sigma_{1}+3, \sigma_{2}+1}^{\psi}\right) \\
& +\left(1-\sum_{l=0}^{3} \rho_{l} \sum_{l=0}^{3} \rho_{l}\right)\left(\varphi_{\sigma_{1}+3, \sigma_{2}+3}^{\psi}-\varphi_{\sigma_{1}+3, \sigma_{2}+2}^{\psi}\right) .
\end{aligned}
$$

We know that $1-\left(\sum_{r=0}^{3} \rho_{r}\right)^{2}=0$. Hence if we take the infinity norm of (43), we obtain

$$
\left\|\varphi_{2 \sigma_{1}, 2 \sigma_{2}}^{\psi+1}-\varphi_{\sigma_{1}, \sigma_{2}}^{\psi}\right\| \leq \delta_{1} \max _{\sigma_{1}, \sigma_{2}}\left\|\varphi_{\sigma_{1}+1, \sigma_{2}}^{\psi}-\varphi_{\sigma_{1}, \sigma_{2}}^{\psi}\right\|+\delta_{1} \max _{\sigma_{1}, \sigma_{2}}\left\|\varphi_{\sigma_{1}, \sigma_{2}+1}^{\psi}-\varphi_{\sigma_{1}, \sigma_{2}}^{\psi}\right\|
$$

where

$$
\delta_{1}=\left|3-\left(\rho_{2}+2 \rho_{1}+3 \rho_{0}\right)\right| .
$$

Similarly, we calculate the following three inequalities:

$$
\begin{aligned}
& \left\|\varphi_{2 \sigma_{1}+1,2 \sigma_{2}}^{\psi+1}-\frac{1}{2}\left(\varphi_{\sigma_{1}, \sigma_{2}}^{\psi}+\varphi_{\sigma_{1}+1, \sigma_{2}}^{\psi}\right)\right\| \\
& \quad \leq \delta_{2} \max _{\sigma_{1}, \sigma_{2}}\left\|\varphi_{\sigma_{1}, \sigma_{2}+1}^{\psi}-\varphi_{\sigma_{1}, \sigma_{2}}^{\psi}\right\|+\delta_{3} \max _{\sigma_{1}, \sigma_{2}}\left\|\varphi_{\sigma_{1}+1, \sigma_{2}}^{\psi}-\varphi_{\sigma_{1}, \sigma_{2}}^{\psi}\right\|,
\end{aligned}
$$

where

$$
\delta_{2}=\left|3-\left(\rho_{2}+2 \rho_{1}+3 \rho_{0}\right)\right|,
$$




$$
\begin{aligned}
& \delta_{3}=\left|\frac{5}{2}-\left(\chi_{2}+2 \chi_{1}+3 \chi_{0}\right)\right|, \\
& \left\|\varphi_{2 \sigma_{1}, 2 \sigma_{2}+1}^{\psi+1}-\frac{1}{2}\left(\varphi_{\sigma_{1}, \sigma_{2}}^{\psi}+\varphi_{\sigma_{1}, \sigma_{2}+1}^{\psi}\right)\right\| \\
& \leq \delta_{3} \max _{\sigma_{1}, \sigma_{2}}\left\|\varphi_{\sigma_{1}, \sigma_{2}+1}^{\psi}-\varphi_{\sigma_{1}, \sigma_{2}}^{\psi}\right\|+\delta_{2} \max _{\sigma_{1}, \sigma_{2}}\left\|\varphi_{\sigma_{1}+1, \sigma_{2}}^{\psi}-\varphi_{\sigma_{1}, \sigma_{2}}^{\psi}\right\|,
\end{aligned}
$$

where

$$
\begin{aligned}
\delta_{3} & =\left|\frac{5}{2}-\left(\chi_{2}+2 \chi_{1}+3 \chi_{0}\right)\right|, \\
\delta_{2} & =\left|3-\left(\rho_{2}+2 \rho_{1}+3 \rho_{0}\right)\right|, \\
\left\|\varphi_{2 \sigma_{1}+1,2 \sigma_{2}+1}^{\psi+1}-\frac{1}{4}\left(\varphi_{\sigma_{1}, \sigma_{2}}^{\psi}+\varphi_{\sigma_{1}+1, \sigma_{2}}^{\psi}+\varphi_{\sigma_{1}, \sigma_{2}+1}^{\psi}+\varphi_{\sigma_{1}+1, \sigma_{2}+1}^{\psi}\right)\right\| & \leq \delta_{4} \max _{\sigma_{1}, \sigma_{2}}\left\|\varphi_{\sigma_{1}, \sigma_{2}+1}^{\psi}-\varphi_{\sigma_{1}, \sigma_{2}}^{\psi}\right\|+\delta_{4} \max _{\sigma_{1}, \sigma_{2}}\left\|\varphi_{\sigma_{1}+1, \sigma_{2}}^{\psi}-\varphi_{\sigma_{1}, \sigma_{2}}^{\psi}\right\|,
\end{aligned}
$$

where

$$
\delta_{4}=\left|\frac{5}{2}-\left(\chi_{2}+2 \chi_{1}+3 \chi_{0}\right)\right|
$$

By combining inequalities in (44)-(46), we get

$$
\left\|\varphi^{\psi+1}-\varphi^{\psi}\right\|_{\infty} \leq \delta\left\{\max _{\sigma_{1}, \sigma_{2}}\left\|\varphi_{\sigma_{1}+1, \sigma_{2}}^{\psi}-\varphi_{\sigma_{1}, \sigma_{2}}^{\psi}\right\|+\max _{\sigma_{1}, \sigma_{2}}\left\|\varphi_{\sigma_{1}, \sigma_{2}+1}^{\psi}-\varphi_{\sigma_{1}, \sigma_{2}}^{\psi}\right\|\right\}
$$

where

$$
\delta=\max \left\{\delta_{1}, \delta_{2}, \delta_{3}, \delta_{4}\right\}
$$

This implies that

$$
\left\|\varphi^{\psi+1}-\varphi^{\psi}\right\|_{\infty} \leq \delta\left\{\max _{\sigma_{1}, \sigma_{2}}\left\|\varphi_{\sigma_{1}+1, \sigma_{2}}^{0}-\varphi_{\sigma_{1}, \sigma_{2}}^{0}\right\|+\max _{\sigma_{1}, \sigma_{2}}\left\|\varphi_{\sigma_{1}, \sigma_{2}+1}^{0}-\varphi_{\sigma_{1}, \sigma_{2}}^{0}\right\|\right\} .
$$

This completes the proof.

\subsection{The deviation of first order GBDDRS}

In this part of the article, we first introduce the inequalities to compute the deviation between two consecutive points at the $(\psi+1)$ th refinement level then we introduce the inequalities to compute the deviation between the $\psi$ th and $(\psi+1)$ th level of meshes produced by first order GBDDRS.

Lemma 9 Let $E^{0}=\left\{\left(\sigma_{1}, \sigma_{2}, E_{\sigma_{1}, \sigma_{2}}^{0}\right): \sigma_{1}, \sigma_{2} \in \mathbb{Z}\right\}$ be an initial mesh and $E^{\psi+1}=\left\{\left(\frac{\sigma_{1}}{2^{\psi+1}}, \frac{\sigma_{2}}{2^{\psi+1}}\right.\right.$, $\left.\left.E_{\sigma_{1}, \sigma_{2}}^{\psi+1}\right): \sigma_{1}, \sigma_{2} \in \mathbb{Z}, \psi \in \mathbb{N}\right\}$ be a mesh produced by the first order GBDDRS at the $(\psi+1)$ th refinement level. If $J=0$ and we fix $m=3$, then the deviations between two consecutive points at the $(\psi+1)$ th refinement step are

$$
\left\{\begin{array}{l}
\max _{\sigma_{1}, \sigma_{2}}\left\|E_{\sigma_{1}+1, \sigma_{2}}^{\psi+1}-E_{\sigma_{1}, \sigma_{2}}^{\psi+1}\right\| \leq(G)^{\psi+1} \max _{\sigma_{1}, \sigma_{2}}\left\|E_{\sigma_{1}+1, \sigma_{2}}^{0}-E_{\sigma_{1}, \sigma_{2}}^{0}\right\|, \\
\max _{\sigma_{1}, \sigma_{2}}\left\|E_{\sigma_{1}, \sigma_{2}+1}^{\psi+1}-E_{\sigma_{1}, \sigma_{2}}^{\psi+1}\right\| \leq(G)^{\psi+1} \max _{\sigma_{1}, \sigma_{2}}\left\|E_{\sigma_{1}, \sigma_{2}+1}^{0}-E_{\sigma_{1}, \sigma_{2}}^{0}\right\|,
\end{array}\right.
$$


where

$$
\begin{aligned}
& G=\max _{\theta}\left\{G_{\theta}: \theta=1,2,3,4\right\}, \\
& \left\{\begin{array}{l}
G_{1}=2^{2}\left|\sum_{l=0}^{2}\left(\sum_{q=0}^{l}\left(\rho_{l-q}-\chi_{l-q}\right)\right)\right| \sum_{t=0}^{2}\left|\sum_{u=0}^{t}\left\{(2 u+1) \rho_{t-u}-(2 u+2) \chi_{t-u}\right\}\right|, \\
G_{2}=2^{2}\left|\sum_{l=0}^{2}\left(\sum_{q=0}^{l}\left(\rho_{l-q}-\chi_{l-q}\right)\right)\right| \sum_{t=0}^{2}\left|\sum_{u=0}^{t}\left\{(2 u+1) \chi_{t-u}-2 u \rho_{t-u}\right\}\right|, \\
G_{3}=2^{2}\left|\sum_{l=0}^{3}\left(\sum_{q=0}^{l}\left(\chi_{l-q}-\rho_{l-q-1}\right)\right)\right| \sum_{t=0}^{2}\left|\sum_{u=0}^{t}\left\{(2 u+1) \rho_{t-u}-(2 u+2) \chi_{t-u}\right\}\right|, \\
G_{4}=2^{2}\left|\sum_{l=0}^{3}\left(\sum_{q=0}^{l}\left(\chi_{l-q}-\rho_{l-q-1}\right)\right)\right| \sum_{t=0}^{2}\left|\sum_{u=0}^{t}\left\{(2 u+1) \chi_{t-u}-2 u \rho_{t-u}\right\}\right|,
\end{array}\right.
\end{aligned}
$$

and

$$
J=\sum_{t=0}^{3}\left\{(2 t+1) \chi_{3-t}-2 t \rho_{3-t}\right\} .
$$

Lemma 10 Let $E^{\psi}=\left\{\left(\frac{\sigma_{1}}{2^{\psi}}, \frac{\sigma_{2}}{2^{\psi}}, E_{\sigma_{1}, \sigma_{2}}^{\psi}\right): \sigma_{1}, \sigma_{2} \in \mathbb{Z}, \psi \in \mathbb{N}\right\}$ be a mesh at the $\psi$ th refinement step and $E^{\psi+1}=\left\{\left(\frac{\sigma_{1}}{2^{\psi+1}}, \frac{\sigma_{2}}{2^{\psi+1}}, E_{\sigma_{1}, \sigma_{2}}^{\psi+1}\right): \sigma_{1}, \sigma_{2} \in \mathbb{Z}, \psi \in \mathbb{N}\right\}$ be a mesh produced by the first order GDDBRS at the $(\psi+1)$ th refinement level. If $J_{1}=J_{2}=J_{3}=0$ and we fix $m=3$, then the deviation between two consecutive meshes at the $\psi$ th and $(\psi+1)$ th refinement steps is

$$
\left\|E^{\psi+1}-E^{\psi}\right\|_{\infty} \leq \lambda\left\{\max _{\sigma_{1}, \sigma_{2}}\left\|E_{\sigma_{1}+1, \sigma_{2}}^{\psi}-E_{\sigma_{1}, \sigma_{2}}^{\psi}\right\|+\max _{\sigma_{1}, \sigma_{2}}\left\|E_{\sigma_{1}, \sigma_{2}+1}^{\psi}-E_{\sigma_{1}, \sigma_{2}}^{\psi}\right\|\right\}
$$

where

$$
\begin{aligned}
& \lambda=\max _{\theta}\left\{\lambda_{\theta}: \theta=1,2,3,4\right\}, \\
& \left\{\begin{array}{l}
\lambda_{1}=2^{2}\left|\frac{1}{2}-\left(\rho_{1}+3 \rho_{0}-\chi_{1}-3 \chi_{0}\right)\left(\rho_{2}+2 \rho_{1}+3 \rho_{0}-\chi_{2}-2 \chi_{1}-3 \chi_{0}\right)\right|, \\
\lambda_{2}=2^{2}\left|\frac{5}{2^{3}}-\left(\rho_{2}+2 \rho_{1}+3 \rho_{0}-\chi_{2}-2 \chi_{1}-3 \chi_{0}\right)\left(\chi_{2}+3 \chi_{1}+6 \chi_{0}-\rho_{1}-3 \rho_{0}\right)\right|, \\
\lambda_{3}=2^{2}\left|\frac{1}{2}-\left(\rho_{1}+3 \rho_{0}-\chi_{1}-3 \chi_{0}\right)\left(\chi_{3}+2 \chi_{2}+3 \chi_{1}+4 \chi_{0}-\rho_{2}-2 \rho_{1}-3 \rho_{0}\right)\right|, \\
\lambda_{4}=2^{2}\left|\frac{5}{2^{3}}-\left(\chi_{2}+3 \chi_{1}+6 \chi_{0}-\rho_{1}-3 \rho_{0}\right)\left(\chi_{3}+2 \chi_{2}+3 \chi_{1}+4 \chi_{0}-\rho_{2}-2 \rho_{1}-3 \rho_{0}\right)\right|,
\end{array}\right.
\end{aligned}
$$

and

$$
\left\{\begin{aligned}
J_{1}= & \frac{-1}{2^{2}}+\left(\rho_{2}+2 \rho_{1}+3 \rho_{0}-\chi_{2}-2 \chi_{1}-3 \chi_{0}\right)^{2}, \\
J_{2}= & \frac{-1}{2^{2}}+\left(\rho_{2}+2 \rho_{1}+3 \rho_{0}-\chi_{2}-2 \chi_{1}-3 \chi_{0}\right) \\
& \times\left(\chi_{3}+2 \chi_{2}+3 \chi_{1}+4 \chi_{0}-\rho_{2}-2 \rho_{1}-3 \rho_{0}\right), \\
J_{3}= & \frac{-1}{2^{2}}+\left(\chi_{3}+2 \chi_{2}+3 \chi_{1}+4 \chi_{0}-\rho_{2}-2 \rho_{1}-3 \rho_{0}\right)^{2} .
\end{aligned}\right.
$$

\subsection{The deviation of second order GBDDRS}

Here, we first calculate the inequalities to compute the deviation between two consecutive points at the $(\psi+1)$ th refinement level; then we introduce the inequalities to compute the deviation between the $\psi$ th and $(\psi+1)$ th level of meshes produced by second order GBDDRS.

Lemma 11 Let $H^{0}=\left\{\left(\sigma_{1}, \sigma_{2}, H_{\sigma_{1}, \sigma_{2}}^{0}\right): \sigma_{1}, \sigma_{2} \in \mathbb{Z}\right\}$ be an initial mesh and $H^{\psi+1}=\left\{\left(\frac{\sigma_{1}}{2^{\psi+1}}\right.\right.$, $\left.\left.\frac{\sigma_{2}}{2^{\psi+1}}, H_{\sigma_{1}, \sigma_{2}}^{\psi+1}\right): \sigma_{1}, \sigma_{2} \in \mathbb{Z}, \psi \in \mathbb{N}\right\}$ be a mesh produced by the second order GBDDRS at the 
$(\psi+1)$ th refinement level. If $M=0$ and we fix $m=3$, then the deviations between two consecutive points at the $(\psi+1)$ th refinement step are

$$
\left\{\begin{array}{l}
\max _{\sigma_{1}, \sigma_{2}}\left\|H_{\sigma_{1}+1, \sigma_{2}}^{\psi+1}-H_{\sigma_{1}, \sigma_{2}}^{\psi+1}\right\| \leq(L)^{\psi+1} \max _{\sigma_{1}, \sigma_{2}}\left\|H_{\sigma_{1}+1, \sigma_{2}}^{0}-H_{\sigma_{1}, \sigma_{2}}^{0}\right\|, \\
\max _{\sigma_{1}, \sigma_{2}}\left\|H_{\sigma_{1}, \sigma_{2}+1}^{\psi+1}-H_{\sigma_{1}, \sigma_{2}}^{\psi+1}\right\| \leq(L)^{\psi+1} \max _{\sigma_{1}, \sigma_{2}}\left\|H_{\sigma_{1}, \sigma_{2}+1}^{0}-H_{\sigma_{1}, \sigma_{2}}^{0}\right\|,
\end{array}\right.
$$

where

$$
\begin{aligned}
& L=\max _{\theta}\left\{L_{\theta}: \theta=1,2,3,4\right\}, \\
& \qquad \begin{aligned}
L_{1}= & 2^{4}\left|\sum_{l=0}^{2}\left(\sum_{q=0}^{l}\left\{(2 q+1) \rho_{l-q}-(2 q+2) \chi_{l-q}\right\}\right)\right| \\
& \times \sum_{t=0}^{1}\left|\sum_{u=0}^{t}(u+1)\left\{(2 u+1) \rho_{t-u}-(2 u+3) \chi_{t-u}\right\}\right|, \\
L_{2}= & 2^{4}\left|\sum_{l=0}^{2}\left(\sum_{q=0}^{l}\left\{(2 q+1) \rho_{l-q}-(2 q+2) \chi_{l-q}\right\}\right)\right| \\
& \times \sum_{t=0}^{2}\left|\sum_{u=0}^{t}(2 u+1)\left\{(u+1) \chi_{t-u}-u \rho_{t-u}\right\}\right|, \\
L_{3}= & 2^{4}\left|\sum_{l=0}^{2}\left(\sum_{q=0}^{l}\left\{(2 q+1) \chi_{l-q}-2 q \rho_{l-q}\right\}\right)\right| \\
& \times \sum_{t=0}^{1}\left|\sum_{u=0}^{t}(u+1)\left\{(2 u+1) \rho_{t-u}-(2 u+3) \chi_{t-u}\right\}\right|, \\
L_{4}= & 2^{4}\left|\sum_{l=0}^{2}\left(\sum_{q=0}^{l}\left\{(2 q+1) \chi_{l-q}-2 q \rho_{l-q}\right\}\right)\right| \\
& \times \sum_{t=0}^{2}\left|\sum_{u=0}^{t}(2 u+1)\left\{(u+1) \chi_{t-u}-u \rho_{t-u}\right\}\right|,
\end{aligned}
\end{aligned}
$$

and

$$
M=\sum_{\sigma=1}^{3} \sigma\left\{(2 \sigma+1) \chi_{3-\sigma}-(2 \sigma-1) \rho_{3-\sigma}\right\}
$$

Lemma 12 Let $H^{\psi}=\left\{\left(\frac{\sigma_{1}}{2^{\psi}}, \frac{\sigma_{2}}{2^{\psi}}, H_{\sigma_{1}, \sigma_{2}}^{\psi}\right): \sigma_{1}, \sigma_{2} \in \mathbb{Z}, \psi \in \mathbb{N}\right\}$ be a mesh at the $\psi$ th refinement step and $H^{\psi+1}=\left\{\left(\frac{\sigma_{1}}{2^{\psi+1}}, \frac{\sigma_{2}}{2^{\psi+1}}, H_{\sigma_{1}, \sigma_{2}}^{\psi+1}\right): \sigma_{1}, \sigma_{2} \in \mathbb{Z}, \psi \in \mathbb{N}\right\}$ be a mesh produced by the second order GDDBRS at the $(\psi+1)$ th refinement level. If $M_{1}=M_{2}=M_{3}=0$ and we fix $m=3$, then the deviation between two consecutive meshes at the $\psi$ th and $(\psi+1)$ th refinement steps is

$$
\left\|H^{\psi+1}-H^{\psi}\right\|_{\infty} \leq \eta\left\{\max _{\sigma_{1}, \sigma_{2}}\left\|H_{\sigma_{1}+1, \sigma_{2}}^{\psi}-H_{\sigma_{1}, \sigma_{2}}^{\psi}\right\|+\max _{\sigma_{1}, \sigma_{2}}\left\|H_{\sigma_{1}, \sigma_{2}+1}^{\psi}-H_{\sigma_{1}, \sigma_{2}}^{\psi}\right\|\right\},
$$

where

$$
\begin{aligned}
& \eta=\max _{\theta}\left\{\eta_{\theta}: \theta=1,2,3,4\right\}, \\
& \left\{\begin{array}{l}
\eta_{1}=2^{4}\left|\frac{1}{2^{3}}-\left(\rho_{2}+4 \rho_{1}+9 \rho_{0}-2 \chi_{2}-6 \chi_{1}-12 \chi_{0}\right)\left(\rho_{1}+5 \rho_{0}-2 \chi_{1}-8 \chi_{0}\right)\right|, \\
\eta_{2}=2^{4}\left|\frac{3}{2^{5}}-\left(\chi_{1}+5 \chi_{0}-2 \rho_{0}\right)\left(\rho_{2}+4 \rho_{1}+9 \rho_{0}-2 \chi_{2}-6 \chi_{1}-12 \chi_{0}\right)\right|, \\
\eta_{3}=2^{4}\left|\frac{1}{2^{3}}-\left(\rho_{1}+5 \rho_{0}-2 \chi_{1}-8 \chi_{0}\right)\left(\chi_{2}+4 \chi_{1}+9 \chi_{0}-2 \rho_{1}-6 \rho_{0}\right)\right|, \\
\eta_{4}=2^{4}\left|\frac{3}{2^{5}}-\left(\chi_{1}+5 \chi_{0}-2 \rho_{0}\right)\left(\chi_{2}+4 \chi_{1}+9 \chi_{0}-2 \rho_{1}-6 \rho_{0}\right)\right|,
\end{array}\right.
\end{aligned}
$$


and

$$
\left\{\begin{aligned}
M_{1}= & \frac{-1}{2^{4}}+\left(\rho_{2}+4 \rho_{1}+9 \rho_{0}-2 \chi_{2}-6 \chi_{1}-12 \chi_{0}\right)^{2} \\
M_{2}= & \frac{-1}{2^{4}}+\left(\rho_{2}+4 \rho_{1}+9 \rho_{0}-2 \chi_{2}-6 \chi_{1}-12 \chi_{0}\right) \\
& \times\left(\chi_{2}+4 \chi_{1}+9 \chi_{0}-2 \rho_{1}-6 \rho_{0}\right), \\
M_{3}= & \frac{-1}{2^{4}}+\left(\chi_{2}+4 \chi_{1}+9 \chi_{0}-2 \rho_{1}-6 \rho_{0}\right)^{2} .
\end{aligned}\right.
$$

\subsection{Generalization of Lemmas 7-12}

In this section, we generalize Lemmas 7-12 for an arbitrary $m$. Thus, we get the following lemmas for the $(m+1)^{2}$-point tensor product binary scheme and its corresponding GBDDRSs.

Lemma 13 (Generalization of Lemma 7 for an arbitrary $m)$ Let $\varphi^{0}=\left\{\left(\sigma_{1}, \sigma_{2}, \varphi_{\sigma_{1}, \sigma_{2}}^{0}\right)\right.$ : $\left.\sigma_{1}, \sigma_{2} \in \mathbb{Z}\right\}$ be an initial mesh and $\varphi^{\psi+1}=\left\{\left(\frac{\sigma_{1}}{2^{\psi+1}}, \frac{\sigma_{2}}{2^{\psi+1}}, \varphi_{\sigma_{1}, \sigma_{2}}^{\psi+1}\right): \sigma_{1}, \sigma_{2} \in \mathbb{Z}, \psi \in \mathbb{N}\right\}$ be a mesh produced by the GTPBRS at the $(\psi+1)$ th refinement level. Then the deviations between two consecutive points at the $(\psi+1)$ th refinement step are

$$
\left\{\begin{array}{l}
\max _{\sigma_{1}, \sigma_{2}}\left\|\varphi_{\sigma_{1}+1, \sigma_{2}}^{\psi+1}-\varphi_{\sigma_{1}, \sigma_{2}}^{\psi+1}\right\| \leq(T)^{\psi+1} \max _{\sigma_{1}, \sigma_{2}}\left\|\varphi_{\sigma_{1}+1, \sigma_{2}}^{0}-\varphi_{\sigma_{1}, \sigma_{2}}^{0}\right\|, \\
\max _{\sigma_{1}, \sigma_{2}}\left\|\varphi_{\sigma_{1}, \sigma_{2}+1}^{\psi+1}-\varphi_{\sigma_{1}, \sigma_{2}}^{\psi+1}\right\| \leq(T)^{\psi+1} \max _{\sigma_{1}, \sigma_{2}}\left\|\varphi_{\sigma_{1}, \sigma_{2}+1}^{0}-\varphi_{\sigma_{1}, \sigma_{2}}^{0}\right\|
\end{array}\right.
$$

where

$$
T=\max _{\theta}\left\{T_{\theta}: \theta=1,2,3,4\right\}
$$

and

$$
\left\{\begin{array}{l}
T_{1}=\left|\sum_{l=0}^{m} \rho_{l}\right| \sum_{t=0}^{m-1}\left|\sum_{u=0}^{t}\left(\rho_{t-u}-\chi_{t-u}\right)\right|, \\
T_{2}=\left|\sum_{l=0}^{m} \rho_{l}\right| \sum_{t=0}^{m}\left|\sum_{u=0}^{t}\left(\chi_{t-u}-\rho_{t-u-1}\right)\right|, \\
T_{3}=\left|\sum_{l=0}^{m} \chi_{l}\right| \sum_{t=0}^{m-1}\left|\sum_{u=0}^{t}\left(\rho_{t-u}-\chi_{t-u}\right)\right|, \\
T_{4}=\left|\sum_{l=0}^{m} \chi_{l}\right| \sum_{t=0}^{m}\left|\sum_{u=0}^{t}\left(\chi_{t-u}-\rho_{t-u-1}\right)\right| .
\end{array}\right.
$$

Lemma 14 (Generalization of Lemma 8 for an arbitrary $m)$ Let $\varphi^{\psi}=\left\{\left(\frac{\sigma_{1}}{2^{\psi}}, \frac{\sigma_{2}}{2^{\psi}}, \varphi_{\sigma_{1}, \sigma_{2}}^{\psi}\right)\right.$ : $\left.\sigma_{1}, \sigma_{2} \in \mathbb{Z}, \psi \in \mathbb{N}\right\}$ be a mesh at the $\psi$ th refinement step and $\varphi^{\psi+1}=\left\{\left(\frac{\sigma_{1}}{2^{\psi+1}}, \frac{\sigma_{2}}{2^{\psi+1}}, \varphi_{\sigma_{1}, \sigma_{2}}^{\psi+1}\right)\right.$ : $\left.\sigma_{1}, \sigma_{2} \in \mathbb{Z}, \psi \in \mathbb{N}\right\}$ be a mesh produced by GTPBRS at the $(\psi+1)$ th refinement level. Then the deviation between two consecutive meshes at the $\psi$ th and $(\psi+1)$ th refinement steps is

$$
\left\|\varphi^{\psi+1}-\varphi^{\psi}\right\|_{\infty} \leq \delta\left\{\max _{\sigma_{1}, \sigma_{2}}\left\|\varphi_{\sigma_{1}+1, \sigma_{2}}^{\psi}-\varphi_{\sigma_{1}, \sigma_{2}}^{\psi}\right\|+\max _{\sigma_{1}, \sigma_{2}}\left\|\varphi_{\sigma_{1}, \sigma_{2}+1}^{\psi}-\varphi_{\sigma_{1}, \sigma_{2}}^{\psi}\right\|\right\},
$$

where

$$
\delta=\max _{\theta}\left\{\delta_{\theta}: \theta=1,2,3,4\right\}
$$


and

$$
\left\{\begin{array}{l}
\delta_{1}=\left|m-\sum_{\sigma=0}^{m-1}(\sigma+1) \rho_{m-1-\sigma}\right|, \\
\delta_{2}=\left|m-\sum_{\sigma=0}^{m-1}(\sigma+1) \rho_{m-1-\sigma}\right|, \\
\delta_{3}=\left|\frac{2 m-1}{2}-\sum_{\sigma=0}^{m-1}(\sigma+1) \chi_{m-1-\sigma}\right|, \\
\delta_{4}=\left|\frac{2 m-1}{2}-\sum_{\sigma=0}^{m-1}(\sigma+1) \chi_{m-1-\sigma}\right| .
\end{array}\right.
$$

Lemma 15 (Generalization of Lemma 9 for an arbitrary $m$ ) Let $E^{0}=\left\{\left(\sigma_{1}, \sigma_{2}, E_{\sigma_{1}, \sigma_{2}}^{0}\right)\right.$ : $\left.\sigma_{1}, \sigma_{2} \in \mathbb{Z}\right\}$ be an initial mesh and $E^{\psi+1}=\left\{\left(\frac{\sigma_{1}}{2^{\psi+1}}, \frac{\sigma_{2}}{2^{\psi+1}}, E_{\sigma_{1}, \sigma_{2}}^{\psi+1}\right): \sigma_{1}, \sigma_{2} \in \mathbb{Z}, \psi \in \mathbb{N}\right\}$ be a mesh produced by the first order GBDDRS at the $(\psi+1)$ th refinement level. If $J=0$, then the deviations between two consecutive points at the $(\psi+1)$ th refinement step are

$$
\left\{\begin{array}{l}
\max _{\sigma_{1}, \sigma_{2}}\left\|E_{\sigma_{1}+1, \sigma_{2}}^{\psi+1}-E_{\sigma_{1}, \sigma_{2}}^{\psi+1}\right\| \leq(G)^{\psi+1} \max _{\sigma_{1}, \sigma_{2}}\left\|E_{\sigma_{1}+1, \sigma_{2}}^{0}-E_{\sigma_{1}, \sigma_{2}}^{0}\right\|, \\
\max _{\sigma_{1}, \sigma_{2}}\left\|E_{\sigma_{1}, \sigma_{2}+1}^{\psi+1}-E_{\sigma_{1}, \sigma_{2}}^{\psi+1}\right\| \leq(G)^{\psi+1} \max _{\sigma_{1}, \sigma_{2}}\left\|E_{\sigma_{1}, \sigma_{2}+1}^{0}-E_{\sigma_{1}, \sigma_{2}}^{0}\right\|,
\end{array}\right.
$$

where

$$
\begin{aligned}
& G=\max _{\theta}\left\{G_{\theta}: \theta=1,2,3,4\right\}, \\
& \left\{\begin{array}{l}
G_{1}=2^{2}\left|\sum_{l=0}^{m-1}\left(\sum_{q=0}^{l}\left(\rho_{l-q}-\chi_{l-q}\right)\right)\right| \sum_{t=0}^{m-1}\left|\sum_{u=0}^{t}\left\{(2 u+1) \rho_{t-u}-(2 u+2) \chi_{t-u}\right\}\right|, \\
G_{2}=2^{2}\left|\sum_{l=0}^{m-1}\left(\sum_{q=0}^{l}\left(\rho_{l-q}-\chi_{l-q}\right)\right)\right| \sum_{t=0}^{m-1}\left|\sum_{u=0}^{t}\left\{(2 u+1) \chi_{t-u}-2 u \rho_{t-u}\right\}\right|, \\
G_{3}=2^{2}\left|\sum_{l=0}^{m}\left(\sum_{q=0}^{l}\left(\chi_{l-q}-\rho_{l-q-1}\right)\right)\right| \sum_{t=0}^{m-1}\left|\sum_{u=0}^{t}\left\{(2 u+1) \rho_{t-u}-(2 u+2) \chi_{t-u}\right\}\right|, \\
G_{4}=2^{2}\left|\sum_{l=0}^{m}\left(\sum_{q=0}^{l}\left(\chi_{l-q}-\rho_{l-q-1}\right)\right)\right| \sum_{t=0}^{m-1}\left|\sum_{u=0}^{t}\left\{(2 u+1) \chi_{t-u}-2 u \rho_{t-u}\right\}\right|,
\end{array}\right.
\end{aligned}
$$

and

$$
J=\sum_{t=0}^{m}\left\{(2 t+1) \chi_{m-t}-2 t \rho_{m-t}\right\}
$$

Lemma 16 (Generalization of Lemma 10 for an arbitrary $m)$ Let $E^{\psi}=\left\{\left(\frac{\sigma_{1}}{2 \psi}, \frac{\sigma_{2}}{2 \psi}, E_{\sigma_{1}, \sigma_{2}}^{\psi}\right)\right.$ : $\left.\sigma_{1}, \sigma_{2} \in \mathbb{Z}, \psi \in \mathbb{N}\right\}$ be a mesh at the $\psi$ th refinement step and $E^{\psi+1}=\left\{\left(\frac{\sigma_{1}}{2^{\psi+1}}, \frac{\sigma_{2}}{2^{\psi+1}}, E_{\sigma_{1}, \sigma_{2}}^{\psi+1}\right)\right.$ : $\left.\sigma_{1}, \sigma_{2} \in \mathbb{Z}, \psi \in \mathbb{N}\right\}$ be a mesh produced by the first order GDDBRS at the $(\psi+1)$ th refinement level. If $J_{1}=J_{2}=J_{3}=0$, then the deviation between two consecutive meshes at the $\psi$ th and $(\psi+1)$ th refinement steps is

$$
\left\|E^{\psi+1}-E^{\psi}\right\|_{\infty} \leq \lambda\left\{\max _{\sigma_{1}, \sigma_{2}}\left\|E_{\sigma_{1}+1, \sigma_{2}}^{\psi}-E_{\sigma_{1}, \sigma_{2}}^{\psi}\right\|+\max _{\sigma_{1}, \sigma_{2}}\left\|E_{\sigma_{1}, \sigma_{2}+1}^{\psi}-E_{\sigma_{1}, \sigma_{2}}^{\psi}\right\|\right\},
$$

where

$$
\lambda=\max _{\theta}\left\{\lambda_{\theta}: \theta=1,2,3,4\right\}
$$




$$
\left\{\begin{aligned}
\lambda_{1}= & \mid(m-1)-\left(\sum_{\sigma=0}^{m-2} \frac{(\sigma+1)(\sigma+2)}{2}\left(\rho_{m-2-\sigma}-\chi_{m-2-\sigma}\right)\right) \\
& \times\left(\sum_{\sigma=0}^{m-1}(\sigma+1)\left(\rho_{m-1-\sigma}-\chi_{m-1-\sigma}\right)\right) \mid, \\
\lambda_{2}= & \mid \frac{2 m-1}{2}-\left(\sum_{\sigma=0}^{m-1} \frac{(\sigma+1)}{2}\left\{(\sigma+2) \chi_{m-1-\sigma}-\sigma \rho_{m-1-\sigma}\right\}\right) \\
& \times\left(\sum_{\sigma=0}^{m-1}(\sigma+1)\left(\rho_{m-1-\sigma}-\chi_{m-1-\sigma}\right)\right) \mid, \\
\lambda_{3}= & \mid(m-1)-\left(\sum_{\sigma=0}^{m-2} \frac{(\sigma+1)(\sigma+2)}{2}\left(\rho_{m-2-\sigma}-\chi_{m-2-\sigma}\right)\right) \\
& \times\left(\sum_{\sigma=0}^{m}\left\{(\sigma+1) \chi_{m-\sigma}-\sigma \rho_{m-\sigma}\right\}\right) \mid, \\
\lambda_{4}= & \mid \frac{2 m-1}{2}-\left(\sum_{\sigma=0}^{m-1} \frac{(\sigma+1)}{2}\left\{(\sigma+2) \chi_{m-1-\sigma}-\sigma \rho_{m-1-\sigma}\right\}\right) \\
& \times\left(\sum_{\sigma=0}^{m}\left\{(\sigma+1) \chi_{m-\sigma}-\sigma \rho_{m-\sigma}\right\}\right) \mid,
\end{aligned}\right.
$$

and

$$
\left\{\begin{array}{l}
J_{1}=\left(\sum_{t=0}^{m} t\left\{\rho_{m-t}-\chi_{m-t}\right\}\right)^{2}-\frac{1}{2^{2}}, \\
J_{2}=\left(\sum_{t=0}^{m} t\left\{\rho_{m-t}-\chi_{m-t}\right\}\right)\left(\sum_{t=0}^{m}\left\{(t+1) \chi_{m-t}-t \rho_{m-t}\right\}\right)-\frac{1}{2^{2}}, \\
J_{3}=\left(\sum_{t=0}^{m}\left\{(t+1) \chi_{m-t}-t \rho_{m-t}\right\}\right)^{2}-\frac{1}{2^{2}} .
\end{array}\right.
$$

Lemma 17 (Generalization of Lemma 11 for an arbitrary $m$ ) Let $H^{0}=\left\{\left(\sigma_{1}, \sigma_{2}, H_{\sigma_{1}, \sigma_{2}}^{0}\right)\right.$ : $\left.\sigma_{1}, \sigma_{2} \in \mathbb{Z}\right\}$ be an initial mesh and $H^{\psi+1}=\left\{\left(\frac{\sigma_{1}}{2^{\psi+1}}, \frac{\sigma_{2}}{2^{\psi+1}}, H_{\sigma_{1}, \sigma_{2}}^{\psi+1}\right): \sigma_{1}, \sigma_{2} \in \mathbb{Z}, \psi \in \mathbb{N}\right\}$ be a mesh produced by the second order GBDDRS at the $(\psi+1)$ th refinement level. If $M=0$, then the deviations between two consecutive points at the $(\psi+1)$ th refinement step are

$$
\left\{\begin{array}{l}
\max _{\sigma_{1}, \sigma_{2}}\left\|H_{\sigma_{1}+1, \sigma_{2}}^{\psi+1}-H_{\sigma_{1}, \sigma_{2}}^{\psi+1}\right\| \leq(L)^{\psi+1} \max _{\sigma_{1}, \sigma_{2}}\left\|H_{\sigma_{1}+1, \sigma_{2}}^{0}-H_{\sigma_{1}, \sigma_{2}}^{0}\right\|, \\
\max _{\sigma_{1}, \sigma_{2}}\left\|H_{\sigma_{1}, \sigma_{2}+1}^{\psi+1}-H_{\sigma_{1}, \sigma_{2}}^{\psi+1}\right\| \leq(L)^{\psi+1} \max _{\sigma_{1}, \sigma_{2}}\left\|H_{\sigma_{1}, \sigma_{2}+1}^{0}-H_{\sigma_{1}, \sigma_{2}}^{0}\right\|,
\end{array}\right.
$$

where

$$
\begin{aligned}
& L=\max _{\theta}\left\{L_{\theta}: \theta=1,2,3,4\right\}, \\
& \qquad \begin{aligned}
L_{1}= & 2^{4}\left|\sum_{l=0}^{m-1}\left(\sum_{q=0}^{l}\left\{(2 q+1) \rho_{l-q}-(2 q+2) \chi_{l-q}\right\}\right)\right| \\
& \times \sum_{t=0}^{m-2}\left|\sum_{u=0}^{t}(u+1)\left\{(2 u+1) \rho_{t-u}-(2 u+3) \chi_{t-u}\right\}\right|, \\
L_{2}= & 2^{4}\left|\sum_{l=0}^{m-1}\left(\sum_{q=0}^{l}\left\{(2 q+1) \rho_{l-q}-(2 q+2) \chi_{l-q}\right\}\right)\right| \\
& \times \sum_{t=0}^{m-1}\left|\sum_{u=0}^{t}(2 u+1)\left\{(u+1) \chi_{t-u}-u \rho_{t-u}\right\}\right|, \\
L_{3}= & 2^{4}\left|\sum_{l=0}^{m-1}\left(\sum_{q=0}^{l}\left\{(2 q+1) \chi_{l-q}-2 q \rho_{l-q}\right\}\right)\right| \\
& \times \sum_{t=0}^{m-2}\left|\sum_{u=0}^{t}(u+1)\left\{(2 u+1) \rho_{t-u}-(2 u+3) \chi_{t-u}\right\}\right|, \\
L_{4}= & 2^{4}\left|\sum_{l=0}^{m-1}\left(\sum_{q=0}^{l}\left\{(2 q+1) \chi_{l-q}-2 q \rho_{l-q}\right\}\right)\right| \\
& \times \sum_{t=0}^{m-1}\left|\sum_{u=0}^{t}(2 u+1)\left\{(u+1) \chi_{t-u}-u \rho_{t-u}\right\}\right|,
\end{aligned}
\end{aligned}
$$

and

$$
M=\sum_{\sigma=1}^{m} \sigma\left\{(2 \sigma+1) \chi_{m-\sigma}-(2 \sigma-1) \rho_{m-\sigma}\right\} .
$$

Lemma 18 (Generalization of Lemma 12 for an arbitrary $m)$ Let $H^{\psi}=\left\{\left(\frac{\sigma_{1}}{2^{\psi}}, \frac{\sigma_{2}}{2^{\psi}}, H_{\sigma_{1}, \sigma_{2}}^{\psi}\right)\right.$ : $\left.\sigma_{1}, \sigma_{2} \in \mathbb{Z}, \psi \in \mathbb{N}\right\}$ be a mesh at the $\psi$ th refinement step and $H^{\psi+1}=\left\{\left(\frac{\sigma_{1}}{2^{\psi+1}}, \frac{\sigma_{2}}{2^{\psi+1}}, H_{\sigma_{1}, \sigma_{2}}^{\psi+1}\right)\right.$ : 
$\left.\sigma_{1}, \sigma_{2} \in \mathbb{Z}, \psi \in \mathbb{N}\right\}$ be a mesh produced by the second order GDDBRS at the $(\psi+1)$ th refinement level. If $M_{1}=M_{2}=M_{3}=0$, then the deviation between two consecutive meshes at the $\psi$ th and $(\psi+1)$ th refinement steps is

$$
\left\|H^{\psi+1}-H^{\psi}\right\|_{\infty} \leq \eta\left\{\max _{\sigma_{1}, \sigma_{2}}\left\|H_{\sigma_{1}+1, \sigma_{2}}^{\psi}-H_{\sigma_{1}, \sigma_{2}}^{\psi}\right\|+\max _{\sigma_{1}, \sigma_{2}}\left\|H_{\sigma_{1}, \sigma_{2}+1}^{\psi}-H_{\sigma_{1}, \sigma_{2}}^{\psi}\right\|\right\},
$$

where

$$
\begin{aligned}
& \eta \max _{\theta}\left\{\eta_{\theta}: \theta=1,2,3,4\right\}, \\
& \left\{\begin{aligned}
\eta_{1}= & \mid(m-1)-\left(\sum_{\sigma=0}^{m-1}(\sigma+1)\left\{(\sigma+1) \rho_{m-1-\sigma}-(\sigma+2) \chi_{m-1-\sigma}\right\}\right) \\
& \times\left(\sum_{\sigma=0}^{m-2}\left\{(4 \sigma+1) \rho_{m-2-\sigma}-(6 \sigma+2) \chi_{m-2-\sigma}\right\}\right) \mid, \\
\eta_{2}= & \mid \frac{2 m-3}{2}-\left(\sum_{\sigma=0}^{m-1}(\sigma+1)\left\{(\sigma+1) \rho_{m-1-\sigma}-(\sigma+2) \chi_{m-1-\sigma}\right\}\right) \\
& \times\left(\sum_{\sigma=0}^{m-2}\left\{(4 \sigma+1) \chi_{m-2-\sigma}-6 \sigma \rho_{m-2-\sigma}\right\}\right) \mid, \\
\eta_{3}= & \mid(m-1)-\left(\sum_{\sigma=0}^{m-1}(\sigma+1)\left\{(\sigma+1) \chi_{m-1-\sigma}-\sigma \rho_{m-1-\sigma}\right\}\right) \\
& \times\left(\sum_{\sigma=0}^{m-2}\left\{(4 \sigma+1) \rho_{m-2-\sigma}-(6 \sigma+2) \chi_{m-2-\sigma}\right\}\right) \mid, \\
\eta_{4}= & \mid \frac{2 m-3}{2}-\left(\sum_{\sigma=0}^{m-1}(\sigma+1)\left\{(\sigma+1) \chi_{m-1-\sigma}-\sigma \rho_{m-1-\sigma}\right\}\right) \\
& \times\left(\sum_{\sigma=0}^{m-2}\left\{(4 \sigma+1) \chi_{m-2-\sigma}-6 \sigma \rho_{m-2-\sigma}\right\}\right) \mid,
\end{aligned}\right.
\end{aligned}
$$

and

$$
\left\{\begin{aligned}
M_{1}= & \left(\sum_{t=0}^{m}\left\{\left(t^{2} \rho_{m-t}-t(t+1) \chi_{m-t}\right\}\right)^{2}-\frac{1}{2^{4}},\right. \\
M_{2}= & \left(\sum_{t=0}^{m}\left\{\left(t^{2} \rho_{m-t}-t(t+1) \chi_{m-t}\right\}\right)\right. \\
& \times\left(\sum_{t=0}^{m}\left\{t^{2} \chi_{m-t}-t(t-1) \rho_{m-t}\right\}\right)-\frac{1}{2^{4}}, \\
M_{3}= & \left(\sum_{t=0}^{m}\left\{t^{2} \chi_{m-t}-t(t-1) \rho_{m-t}\right\}\right)^{2}-\frac{1}{2^{4}} .
\end{aligned}\right.
$$

\section{The smoothness of the models produced by the GTPBRS}

Let $\left\{\Gamma_{[0, n]^{2}}^{\kappa}, \kappa=0,1,2\right\}$ be the set of $C^{\kappa}$-continuous functions on a closed and bounded interval $[0, n] \times[0, n]$. A bivariate function is called $C^{\kappa}$-continuous if its $\kappa$ th order partial derivatives are continuous. The common class of continuous function is $C=C^{0}$. The graphs of $C^{2}$-continuous functions are smooth, while the graphs of $C^{0}$-continuous functions are comparatively rough. In Fig. 2, red bullets and red lines represents initial control points and initial meshes. Figure 2(a) and Fig. 2(b) show the $C^{0}$-continuous and $C^{2}$ continuous meshes produced by the bivariate schemes after three refinement levels, respectively. In this section, we give some basic results for $C^{\kappa}$-smoothness of the GTPBRS, where $\kappa=0,1,2$.

Theorem 1 Let $\varphi^{0}=\left\{\left(\sigma_{1}, \sigma_{2}, \varphi_{\sigma_{1}, \sigma_{2}}^{0}\right): \sigma_{1}, \sigma_{2} \in \mathbb{Z}\right\}$ be an initial mesh and $\varphi^{\psi+1}=\left\{\left(\frac{\sigma_{1}}{2^{\psi+1}}\right.\right.$, $\left.\left.\frac{\sigma_{2}}{2^{\psi+1}}, \varphi_{\sigma_{1}, \sigma_{2}}^{\psi+1}\right): \sigma_{1}, \sigma_{2} \in \mathbb{Z}, \psi \in \mathbb{N}\right\}$ be a mesh produced by the GTPBRS after $(\psi+1)$ refinement steps. If $T<1$ then $\lim _{\psi \rightarrow \infty} \varphi^{\psi}=\varphi \in \Gamma_{[0, n]^{2}}=\Gamma_{[0, n]^{2}}^{0}$.

Proof We use (66) to get the following result:

$$
\left\|\varphi^{\psi+1}-\varphi^{\psi}\right\|_{\infty} \leq \delta\left\{\max _{\sigma_{1}, \sigma_{2}}\left\|\varphi_{\sigma_{1}+1, \sigma_{2}}^{\psi}-\varphi_{\sigma_{1}, \sigma_{2}}^{\psi}\right\|+\max _{\sigma_{1}, \sigma_{2}}\left|\varphi_{\sigma_{1}, \sigma_{2}+1}^{\psi}-\varphi_{\sigma_{1}, \sigma_{2}}^{\psi}\right|\right\}
$$

where $\delta$ is defined in (67). 


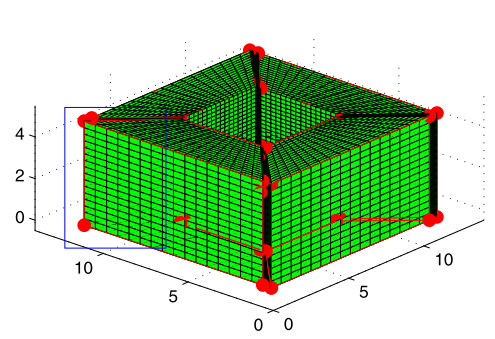

(a)

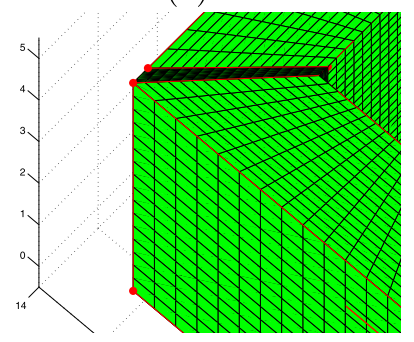

(c)

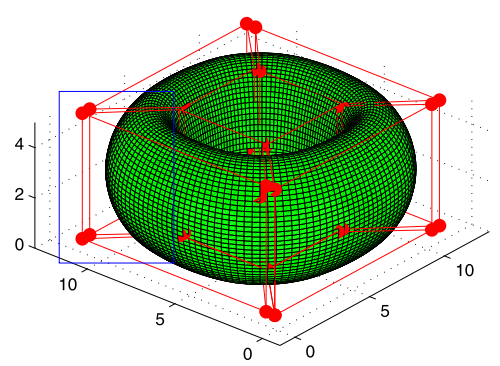

(b)

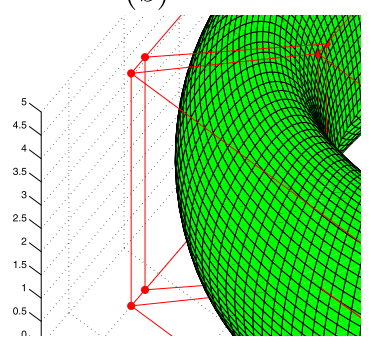

(d)

Figure 2 Surfaces generated by the tensor product schemes. (c)-(d) are the mirror images of the parts inside the blue rectangles of (a)-(b), respectively

Now by using (63), we obtain

$$
\left\|\varphi^{\psi+1}-\varphi^{\psi}\right\|_{\infty} \leq \delta(T)^{\psi}\left\{\max _{\sigma_{1}, \sigma_{2}}\left\|\varphi_{\sigma_{1}+1, \sigma_{2}}^{0}-\varphi_{\sigma_{1}, \sigma_{2}}^{0}\right\|+\max _{\sigma_{1}, \sigma_{2}}\left\|\varphi_{\sigma_{1}, \sigma_{2}+1}^{0}-\varphi_{\sigma_{1}, \sigma_{2}}^{0}\right\|\right\},
$$

where $T$ is defined in (64).

Let $T<1$. This implies that $\left\{\varphi^{\psi}\right\}_{\psi=0}^{\infty}$ is a Cauchy sequence on $\Gamma_{[0, n]^{2}}$. Thus, it is convergent. Therefore,

$$
\lim _{\psi \rightarrow \infty} \varphi^{\psi}=\varphi \in \Gamma_{[0, n]^{2}}
$$

This completes the proof.

Lemma 19 If $E^{0}=\left\{\left(\sigma_{1}, \sigma_{2}, E_{\sigma_{1}, \sigma_{2}}^{0}\right): \sigma_{1}, \sigma_{2} \in \mathbb{Z}\right\}$ is an initial mesh and $E^{\psi+1}=\left\{\left(\frac{\sigma_{1}}{2^{\psi+1}}\right.\right.$, $\left.\left.\frac{\sigma_{2}}{2^{\psi+1}}, E_{\sigma_{1}, \sigma_{2}}^{\psi+1}\right): \sigma_{1}, \sigma_{2} \in \mathbb{Z}, \psi \in \mathbb{N}\right\}$ is a mesh produced by the first order GBDDRS at the $(\psi+1)$ th refinement step. If $J, J_{1}, J_{2}, J_{3}=0$ and $G<1$ then $\lim _{\psi \rightarrow \infty} E^{\psi}=E \in \Gamma_{[0, n]^{2}}=\Gamma_{[0, n]^{2}}^{0}$.

Proof By (73), we have

$$
\left\|E^{\psi+1}-E^{\psi}\right\|_{\infty} \leq \lambda\left\{\max _{\sigma_{1}, \sigma_{2}}\left\|E_{\sigma_{1}+1, \sigma_{2}}^{\psi}-E_{\sigma_{1}, \sigma_{2}}^{\psi}\right\|+\max _{\sigma_{1}, \sigma_{2}}\left\|E_{\sigma_{1}, \sigma_{2}+1}^{\psi}-E_{\sigma_{1}, \sigma_{2}}^{\psi}\right\|\right\}
$$

where $\lambda$ is defined in (74).

Now by using (69) in the above inequality, we get

$$
\left\|E^{\psi+1}-E^{\psi}\right\|_{\infty} \leq \lambda(G)^{\psi}\left\{\max _{\sigma_{1}, \sigma_{2}}\left\|E_{\sigma_{1}+1, \sigma_{2}}^{0}-E_{\sigma_{1}, \sigma_{2}}^{0}\right\|+\max _{\sigma_{1}, \sigma_{2}}\left\|E_{\sigma_{1}, \sigma_{2}+1}^{0}-E_{\sigma_{1}, \sigma_{2}}^{0}\right\|\right\},
$$

where $G$ is defined in (70). 
If $G<1$, then $\left\{E^{\psi}\right\}_{\psi=0}^{\infty}$ is a Cauchy sequence on $\Gamma_{[0, n]^{2}}$ and hence

$$
\lim _{\psi \rightarrow \infty} E^{\psi}=E \in \Gamma_{[0, n]^{2}}
$$

This completes the proof.

Theorem 2 Let $\varphi \in \Gamma_{[0, n]^{2}}$ be the limit function of GTPBRS defined in (1). If J, $J_{1}, J_{2}, J_{3}=0$, and $G<1$, then we have $\varphi \in \Gamma_{[0, n]^{2}}^{1}$.

Proof By Lemma 19, we have $\lim _{\psi \rightarrow \infty} E^{\psi}=E \in \Gamma_{[0, n]^{2}}$. By Lemma 5, we have $E=\varphi^{\prime}$, where $\varphi$ is the limit function of the GTPBRS defined in (1). This implies that $\varphi \in \Gamma_{[0, n]^{2}}^{1}$.

Lemma 20 If $H^{0}=\left\{\left(\sigma_{1}, \sigma_{2}, H_{\sigma_{1}, \sigma_{2}}^{0}\right): \sigma_{1}, \sigma_{2} \in \mathbb{Z}\right\}$ is an initial mesh and $H^{\psi+1}=\left\{\left(\frac{\sigma_{1}}{2^{\psi+1}}\right.\right.$, $\left.\left.\frac{\sigma_{2}}{2^{\psi+1}}, H_{\sigma_{1}, \sigma_{2}}^{\psi+1}\right): \sigma_{1}, \sigma_{2} \in \mathbb{Z}, \psi \in \mathbb{N}\right\}$ is a mesh produced by the second order GBDDRS at the $(\psi+1)$ th refinement step. If $M=M_{1}=M_{2}=M_{3}=0$ and $L<1$ then $\lim _{\psi \rightarrow \infty} H^{\psi}=H \in$ $\Gamma_{[0, n]^{2}}=\Gamma_{[0, n]^{2}}^{0}$.

Proof By (81), we have

$$
\left\|H^{\psi+1}-H^{\psi}\right\|_{\infty} \leq \eta\left\{\max _{\sigma_{1}, \sigma_{2}}\left\|H_{\sigma_{1}+1, \sigma_{2}}^{\psi}-H_{\sigma_{1}, \sigma_{2}}^{\psi}\right\|+\max _{\sigma_{1}, \sigma_{2}}\left\|H_{\sigma_{1}, \sigma_{2}+1}^{\psi}-H_{\sigma_{1}, \sigma_{2}}^{\psi}\right\|\right\}
$$

where $\eta$ is defined in (82).

Now by using (77) in the above expression, we obtain

$$
\left\|H^{\psi+1}-H^{\psi}\right\|_{\infty} \leq \eta(L)^{\psi}\left\{\max _{\sigma_{1}, \sigma_{2}}\left\|H_{\sigma_{1}+1, \sigma_{2}}^{0}-H_{\sigma_{1}, \sigma_{2}}^{0}\right\|+\max _{\sigma_{1}, \sigma_{2}}\left\|H_{\sigma_{1}, \sigma_{2}+1}^{0}-H_{\sigma_{1}, \sigma_{2}}^{0}\right\|\right\}
$$

where $L$ is defined in (78).

If $L<1$, then it follows that $\left\{H^{\psi}\right\}_{\psi=0}^{\infty}$ gives a Cauchy sequence on $\Gamma_{[0, n]^{2}}$ and thus

$$
\lim _{\psi \rightarrow \infty} H^{\psi}=H \in \Gamma_{[0, n]^{2}}
$$

This completes the proof.

Theorem 3 Let $\varphi \in \Gamma_{[0, n]^{2}}$ be the limit function of the GTPBRS defined in (1). If $M, M_{1}, M_{2}$, $M_{3}=0$ and $L<1$, then we have $\varphi \in \Gamma_{[0, n]^{2}}^{2}$.

Proof By Lemma 20, we have $\lim _{\psi \rightarrow \infty} H^{\psi}=H \in \Gamma_{[0, n]^{2}}$. Lemma 6 shows that $H=\varphi^{\prime \prime}$, where $\varphi$ is the limit function of the GTPBRS defined in (1). This implies that $\varphi \in \Gamma_{[0, n]^{2}}^{2}$

\section{Applications of the proposed method}

In this section, we analyze the tensor product schemes of the binary refinement schemes proposed by [21-24] by proposed method. We use Theorem 1 to analyze the $C^{0}$ continuity, Theorem 2 to analyze the $C^{1}$-continuity and Theorem 3 to analyze the $C^{2}$ continuity of the tensor product binary refinement schemes. 
Corollary 1 Let $\varphi_{\sigma_{1}, \sigma_{2}}^{\psi}: \sigma_{1}, \sigma_{2} \in \mathbb{Z}$ be the control points at the $\psi$ th refinement step and $\varphi_{\sigma_{1}, \sigma_{2}}^{\psi+1}$ : $\sigma_{1}, \sigma_{2} \in \mathbb{Z}$ be the refined data/points at the $(\psi+1)$ th refinement step which are refined by using the following interpolatory tensor product scheme of the scheme [21]:

$$
\left\{\begin{array}{l}
\varphi_{2 \sigma_{1}, 2 \sigma_{2}}^{\psi+1}=\sum_{r=0}^{3} \sum_{s=0}^{3} \rho_{r} \rho_{s} \varphi_{\sigma_{1}+r, \sigma_{2}+s}^{\psi} \\
\varphi_{2 \sigma_{1}, 2 \sigma_{2}+1}^{\psi+1}=\sum_{r=0}^{3} \sum_{s=0}^{3} \rho_{r} \chi_{s} \varphi_{\sigma_{1}+r, \sigma_{2}+s}^{\psi} \\
\varphi_{2 \sigma_{1}+1,2 \sigma_{2}}^{\psi+1}=\sum_{r=0}^{3} \sum_{s=0}^{3} \chi_{r} \rho_{s} \varphi_{\sigma_{1}+r, \sigma_{2}+s}^{\psi} \\
\varphi_{2 \sigma_{1}+1,2 \sigma_{2}+1}^{\psi+1}=\sum_{r=0}^{3} \sum_{s=0}^{3} \chi_{r} \chi_{s} \varphi_{\sigma_{1}+r, \sigma_{2}+s}^{\psi}
\end{array}\right.
$$

where

$$
\left\{\begin{array}{l}
\rho_{0}=0, \quad \rho_{1}=1, \quad \rho_{2}=0, \quad \rho_{3}=0, \\
\chi_{0}=-\omega, \quad \chi_{1}=\frac{1}{2}+\omega, \quad \chi_{2}=\frac{1}{2}+\omega, \quad \chi_{3}=-\omega .
\end{array}\right.
$$

Then the surfaces produced by the scheme (85) are $C^{1}$-continuous for parametric interval $\omega \in\left(0, \frac{1}{8}\right)$. But they are not $C^{2}$-continuous.

Proof It is easy to see that

$$
\sum_{r=0}^{3} \sum_{s=0}^{3} \rho_{r} \rho_{s}=\sum_{r=0}^{3} \sum_{s=0}^{3} \rho_{r} \chi_{s}=\sum_{r=0}^{3} \sum_{s=0}^{3} \chi_{r} \rho_{s}=\sum_{r=0}^{3} \sum_{s=0}^{3} \chi_{r} \chi_{s}=1
$$

Also from (65) and (86), we have

$$
\left\{\begin{array}{l}
T_{1}=\left|\sum_{l=0}^{3} \rho_{l}\right| \sum_{t=0}^{2}\left|\sum_{u=0}^{t}\left(\rho_{t-u}-\chi_{t-u}\right)\right|<1, \\
T_{2}=\left|\sum_{l=0}^{3} \rho_{l}\right| \sum_{t=0}^{3}\left|\sum_{u=0}^{t}\left(\chi_{t-u}-\rho_{t-u-1}\right)\right|<1, \\
T_{3}=\left|\sum_{l=0}^{3} \chi_{l}\right| \sum_{t=0}^{2}\left|\sum_{u=0}^{t}\left(\rho_{t-u}-\chi_{t-u}\right)\right|<1, \\
T_{4}=\left|\sum_{l=0}^{3} \chi_{l}\right| \sum_{t=0}^{3}\left|\sum_{u=0}^{t}\left(\chi_{t-u}-\rho_{t-u-1}\right)\right|<1,
\end{array}\right.
$$

for the common interval $\frac{-1}{4}<\omega<\frac{1}{4}$. Hence

$$
T=\max _{\theta}\left\{T_{\theta}: \theta=1,2,3,4\right\}<1
$$

for $\frac{-1}{4}<\omega<\frac{1}{4}$. So by Theorem 1 , the tensor product scheme (85) produces $C^{0}$-continuous surfaces/models when the values of $\omega$ lies between $\frac{-1}{4}$ to $\frac{1}{4}$. Now from (72), (76) and (86), we have

$$
\left\{\begin{array}{l}
J=\sum_{t=0}^{3}\left\{(2 t+1) \chi_{3-t}-2 t \rho_{3-t}\right\}=0 \\
J_{1}=\left(\sum_{t=0}^{3} t\left\{\rho_{3-t}-\chi_{3-t}\right\}\right)^{2}-\frac{1}{2^{2}}=0, \\
J_{2}=\left(\sum_{t=0}^{3} t\left\{\rho_{3-t}-\chi_{3-t}\right\}\right)\left(\sum_{t=0}^{3}\left\{(t+1) \chi_{3-t}-t \rho_{3-t}\right\}\right)-\frac{1}{2^{2}}=0 \\
J_{3}=\left(\sum_{t=0}^{3}\left\{(t+1) \chi_{3-t}-t \rho_{3-t}\right\}\right)^{2}-\frac{1}{2^{2}}=0 .
\end{array}\right.
$$

Also from (71) and (86), we have

$$
\left\{\begin{array}{l}
G_{1}=2^{2}\left|\sum_{l=0}^{m-1}\left(\sum_{q=0}^{l}\left(\rho_{l-q}-\chi_{l-q}\right)\right)\right| \sum_{t=0}^{m-1}\left|\sum_{u=0}^{t}\left\{(2 u+1) \rho_{t-u}-(2 u+2) \chi_{t-u}\right\}\right|<1, \\
G_{2}=2^{2}\left|\sum_{l=0}^{m-1}\left(\sum_{q=0}^{l}\left(\rho_{l-q}-\chi_{l-q}\right)\right)\right| \sum_{t=0}^{m-1}\left|\sum_{u=0}^{t}\left\{(2 u+1) \chi_{t-u}-2 u \rho_{t-u}\right\}\right|<1,
\end{array}\right.
$$


for $\frac{-1}{8}<\omega<\frac{1}{8}$ and

$$
\left\{\begin{array}{l}
G_{3}=2^{2}\left|\sum_{l=0}^{m}\left(\sum_{q=0}^{l}\left(\chi_{l-q}-\rho_{l-q-1}\right)\right)\right| \sum_{t=0}^{m-1}\left|\sum_{u=0}^{t}\left\{(2 u+1) \rho_{t-u}-(2 u+2) \chi_{t-u}\right\}\right|<1, \\
G_{4}=2^{2}\left|\sum_{l=0}^{m}\left(\sum_{q=0}^{l}\left(\chi_{l-q}-\rho_{l-q-1}\right)\right)\right| \sum_{t=0}^{m-1}\left|\sum_{u=0}^{t}\left\{(2 u+1) \chi_{t-u}-2 u \rho_{t-u}\right\}\right|<1,
\end{array}\right.
$$

for $0<\omega<\frac{1}{4}$. Hence $G=\max _{\theta}\left\{G_{\theta}: \theta=1,2,3,4\right\}<1$, for $0<\omega<\frac{1}{8}$. Then, by Theorem 2 , the tensor product scheme (85) produces $C^{1}$-continuous surfaces/models when the values of $\omega$ lie between 0 to $\frac{1}{8}$. By (80), (84) and (86), $M=M_{1}=M_{2}=M_{3} \neq 0$. Hence, by Theorem 3, the models produced by the tensor product scheme (85) are not $C^{2}$-continuous.

Corollary 2 Let $\varphi_{\sigma_{1}, \sigma_{2}}^{\psi}: \sigma_{1}, \sigma_{2} \in \mathbb{Z}$ be the control points at the $\psi$ th refinement step and $\varphi_{\sigma_{1}, \sigma_{2}}^{\psi+1}$ : $\sigma_{1}, \sigma_{2} \in \mathbb{Z}$ be the refined data/points at the $(\psi+1)$ th refinement step which are refined by using the following 9-point approximating tensor product scheme of the scheme [24]:

$$
\left\{\begin{array}{l}
\varphi_{2 \sigma_{1}, 2 \sigma_{2}}^{\psi+1}=\sum_{r=0}^{2} \sum_{s=0}^{2} \rho_{r} \rho_{s} \varphi_{\sigma_{1}+r, \sigma_{2}+s}^{\psi}, \\
\varphi_{2 \sigma_{1}, 2 \sigma_{2}+1}^{\psi+1}=\sum_{r=0}^{2} \sum_{s=0}^{2} \rho_{r} \chi_{s} \varphi_{\sigma_{1}+r, \sigma_{2}+s}^{\psi}, \\
\varphi_{2 \sigma_{1}+1,2 \sigma_{2}}^{\psi+1}=\sum_{r=0}^{2} \sum_{s=0}^{2} \chi_{r} \rho_{s} \varphi_{\sigma_{1}+r, \sigma_{2}+s}^{\psi}, \\
\varphi_{2 \sigma_{1}+1,2 \sigma_{2}+1}^{\psi+1}=\sum_{r=0}^{2} \sum_{s=0}^{2} \chi_{r} \chi_{s} \varphi_{\sigma_{1}+r, \sigma_{2}+s}^{\psi}
\end{array}\right.
$$

where

$$
\left\{\begin{array}{lll}
\rho_{0}=\frac{9}{32}, & \rho_{1}=\frac{22}{32}, & \rho_{2}=\frac{1}{32} \\
\chi_{0}=\frac{1}{32}, & \chi_{1}=\frac{22}{32}, & \chi_{2}=\frac{9}{32} .
\end{array}\right.
$$

Then the models produced by the bivariate scheme (87) are $C^{2}$-continuous.

Corollary 3 Let $\varphi_{\sigma_{1}, \sigma_{2}}^{\psi}: \sigma_{1}, \sigma_{2} \in \mathbb{Z}$ be the control points at the $\psi$ th refinement step and $\varphi_{\sigma_{1}, \sigma_{2}}^{\psi+1}$ : $\sigma_{1}, \sigma_{2} \in \mathbb{Z}$ be the refined data/points at the $(\psi+1)$ th refinement step which are refined by using the following 16-point approximating tensor product scheme of the scheme [23]:

$$
\left\{\begin{array}{l}
\varphi_{2 \sigma_{1}, 2 \sigma_{2}}^{\psi+1}=\sum_{r=0}^{3} \sum_{s=0}^{3} \rho_{r} \rho_{s} \varphi_{\sigma_{1}+r, \sigma_{2}+s}^{\psi}, \\
\varphi_{2 \sigma_{1}, 2 \sigma_{2}+1}^{\psi+1}=\sum_{r=0}^{3} \sum_{s=0}^{3} \rho_{r} \chi_{s} \varphi_{\sigma_{1}+r, \sigma_{2}+s}^{\psi}, \\
\varphi_{2 \sigma_{1}+1,2 \sigma_{2}}^{\psi+1}=\sum_{r=0}^{3} \sum_{s=0}^{3} \chi_{r} \rho_{s} \varphi_{\sigma_{1}+r, \sigma_{2}+s}^{\psi}, \\
\varphi_{2 \sigma_{1}+1,2 \sigma_{2}+1}^{\psi+1}=\sum_{r=0}^{3} \sum_{s=0}^{3} \chi_{r} \chi_{s} \varphi_{\sigma_{1}+r, \sigma_{2}+s}^{\psi}
\end{array}\right.
$$

where

$$
\left\{\begin{array}{l}
\rho_{0}=\frac{1}{16}+3 \mu, \quad \rho_{1}=\frac{5}{8}-5 \mu, \quad \rho_{2}=\frac{5}{16}+\mu, \quad \rho_{3}=\mu \\
\chi_{0}=\mu, \quad \chi_{1}=\frac{5}{16}+\mu, \quad \chi_{2}=\frac{5}{16}+\mu, \quad \chi_{3}=\frac{1}{16}+3 \mu .
\end{array}\right.
$$

Then the shapes produced by the tensor product refinement scheme (88) are $C^{2}$-continuous if we choose $\mu$ from an open interval $\left(\frac{-1}{32}, \frac{3}{32}\right)$. 
Corollary 4 Let $\varphi_{\sigma_{1}, \sigma_{2}}^{\psi}: \sigma_{1}, \sigma_{2} \in \mathbb{Z}$ be the control points at the $\psi$ th refinement step and $\varphi_{\sigma_{1}, \sigma_{2}}^{\psi+1}$ : $\sigma_{1}, \sigma_{2} \in \mathbb{Z}$ be the refined data/points at the $(\psi+1)$ th refinement step which are refined by using the following 36-point approximating tensor product scheme of the scheme [22]:

$$
\left\{\begin{array}{l}
\varphi_{2 \sigma_{1}, 2 \sigma_{2}}^{\psi+1}=\sum_{r=0}^{5} \sum_{s=0}^{5} \rho_{r} \rho_{s} \varphi_{\sigma_{1}+r, \sigma_{2}+s}^{\psi} \\
\varphi_{2 \sigma_{1}, 2 \sigma_{2}+1}^{\psi+1}=\sum_{r=0}^{5} \sum_{s=0}^{5} \rho_{r} \chi_{s} \varphi_{\sigma_{1}+r, \sigma_{2}+s}^{\psi} \\
\varphi_{2 \sigma_{1}+1,2 \sigma_{2}}^{\psi+1}=\sum_{r=0}^{5} \sum_{s=0}^{5} \chi_{r} \rho_{s} \varphi_{\sigma_{1}+r, \sigma_{2}+s}^{\psi} \\
\varphi_{2 \sigma_{1}+1,2 \sigma_{2}+1}^{\psi+1}=\sum_{r=0}^{5} \sum_{s=0}^{5} \chi_{r} \chi_{s} \varphi_{\sigma_{1}+r, \sigma_{2}+s}^{\psi}
\end{array}\right.
$$

where

$$
\left\{\begin{array}{lll}
\rho_{0}=\frac{81}{40,960}, & \rho_{1}=\frac{15,349}{122,880}, & \rho_{2}=\frac{31,927}{61,440}, \\
\rho_{3}=\frac{6719}{20,480}, & \rho_{4}=\frac{3119}{122,880}, & \rho_{5}=\frac{1}{122,880}, \\
\chi_{0}=\frac{1}{122,880}, & \chi_{1}=\frac{3119}{122,880}, & \chi_{2}=\frac{6719}{20,480}, \\
\chi_{3}=\frac{31,927}{61,440}, & \chi_{4}=\frac{15,349}{122,880}, & \chi_{5}=\frac{81}{40,960} .
\end{array}\right.
$$

Then the scheme that is defined in (89) produces the $C^{2}$-continuous limiting shapes.

\section{Summary and conclusion}

In this paper, we have presented some simple conditions to analyze the smoothness of the tensor product version of the $(m+1)$-point binary refinement scheme. The summary of the conditions is given by:

- The GTPBRS defined in (1) is $C^{0}$-continuous if $T=\max _{\theta}\left\{T_{\theta}: \theta=1,2,3,4\right\}<1$, where $T_{\theta}: \theta=1,2,3,4$ are taken from (65).

- The GTPBRS defined in (1) is $C^{1}$-continuous if $G=\max _{\theta}\left\{G_{\theta}: \theta=1,2,3,4\right\}<1$, where $G_{\theta}: \theta=1,2,3,4$ are taken from (71) and $J, J_{1}, J_{2}$ and $J_{3}$ defined in (72) and (76) are zero.

- The GTPBRS defined in (1) is $C^{2}$-continuous if $L=\max _{\theta}\left\{L_{\theta}: \theta=1,2,3,4\right\}<1$, where $L_{\theta}: \theta=1,2,3,4$ are taken from (79) and $M, M_{1}, M_{2}$ and $M_{3}$ defined in (80) and (84) are zero.

Our method of continuity analysis is basically a generalization of the dyadic parametrization method. This method was firstly used by Qu [10-12]. However, he does not provide any general formula. He had just used this method for the analysis of a few particular refinement schemes. The analysis of the bivariate schemes by our method is quite different from the Laurent polynomial method. Because, if the complexity of a refinement scheme is large, then the factorizations of its Laurent polynomial and norms of its mask is difficult to calculate. And in surface case, the computational cost of the Laurent polynomial method is greatly increased. On the other hand, our method provides some simple inequalities which requires mask of that particular scheme.

Our method is superior over other methods because:

- The implementation of our method is very simple and easy even when complexity of the scheme is large.

- Our method is very efficient.

- The formulas involved in our method are of explicit type.

- Our method just requires the mask of the refinement schemes as input.

- Our formulas are free from polynomial algebraic operations. 


\section{Acknowledgements}

We thank the anonymous editors and reviewers for their extensive reading of our manuscript and their many insightful comments and suggestions.

\section{Funding}

Not available.

\section{Availability of data and materials}

Data sharing not applicable to this article as no datasets were generated or analysed during the current study.

\section{Competing interests}

The authors declare that they have no competing interests.

\section{Authors' contributions}

The authors have contributed equally to this manuscript. They read and approved the final manuscript.

\section{Author details}

'Department of Mathematics, The Government Sadiq College Women University Bahawalpur, 63100 Bahawalpur, Pakistan. ${ }^{2}$ Department of Mathematics, The Islamia University of Bahawalpur, 63100 Bahawalpur, Pakistan. ${ }^{3}$ Department of Mathematics, Cankaya University, 06530 Ankara, Turkey. ${ }^{4}$ Institute of Space Sciences, Magurele, 077125 Bucharest, Romania. ${ }^{5}$ Department of Mathematics, Huzhou University, 313000 Huzhou, P.R. China.

\section{Publisher's Note}

Springer Nature remains neutral with regard to jurisdictional claims in published maps and institutional affiliations.

Received: 14 July 2020 Accepted: 9 March 2021 Published online: 22 March 2021

\section{References}

1. Agarwal, P., Tariboon, J., Ntouyas, S.K.: Some generalized Riemann-Liouville $k$-fractional integral inequalities. J. Inequal. Appl. 2016, 122 (2016). https://doi.org/10.1186/s13660-016-1067-3

2. Zhang, X., Agarwal, P., Liu, Z., Peng, H., You, F., Zhu, Y.: Existence and uniqueness of solutions for stochastic differential equations of fractional-order $q>1$ with finite delays. Adv. Differ. Equ. 2017, 123 (2017). https://doi.org/10.1186/s13662-017-1169-3

3. Saoudi, K., Agarwal, P., Kumam, G.A., Thounthong, P.: The Nehari manifold for a boundary value problem involving Riemann-Liouville fractional derivative. Adv. Differ. Equ. 2018, 263 (2018). https://doi.org/10.1186/s13662-018-1722-8

4. Sitho, S., Ntouyas, S.K., Agarwal, P., Tariboon, J.: Noninstantaneous impulsive inequalities via conformable fractional calculus. J. Inequal. Appl. 2018, 261 (2018). https://doi.org/10.1186/s13660-018-1855-z

5. Jain, S., Mehrez, K., Baleanu, D., Agarwal, P.: Certain Hermite-Hadamard inequalities for logarithmically convex functions with applications. Mathematics 7(2), 163 (2019). https://doi.org/10.3390/math7020163

6. Tomar, M., Agarwal, P., Choi, J.: Hermite-Hadamard type inequalities for generalized convex functions on fractal sets style. Bol. Soc. Parana. Mat. 38(1), 101-116 (2020)

7. Dyn, N.: Analysis of convergence and smoothnoess by the formulasiam of Laurent polynomials. In: Iske, A., Quak, E., Floater, M.S. (eds.) Tutorials on Multiresolution in Geometric Modelling, pp. 51-68 (Chap. 3). Springer, Berlin (2002)

8. Levin, D.: Using Laurent polynomial representation for the analysis of non-uniform binary subdivision schemes. Adv. Comput. Math. 11, 41-54 (1999)

9. Dyn, N., Wallner, J.: Convergence and $C^{1}$ analysis of subdivisi on schemes on manifolds by proximity. Comput. Aided Geom. Des. 22, 593-622 (2005)

10. Qu, R.: Recursive subdivision algorithms for curve and surface design. Ph.D. Thesis, Department of Mathematics and Statistics, Brunei University, Uxbridge, Middlesex, Britain (1990)

11. Qu, R., Gregory, J.A.: A 10-point interpolatery recursive subdivision algorithms for the generation of parametric surfaces. TR/01/91, Department of Mathematics and Statistics, Brunei University, Uxbridge, Middlesex, Britain (1991)

12. Qu, R.: An interpolatry subdivision algorithms for surface over orbitrary triangulations. Department of Mathematics and Statistics, Brunel University, Uxbridge, Middlesex, Britain

13. Dyn, N., Gregory, A., Levin, D.: Analysis of uniform binary subdivision schemes for curve design. Constr. Approx. 7 , 127-147 (1991)

14. Dyn, N., Gregory, J.A., Levin, D.: A 4-point interpolatory subdivision scheme for curve design. Comput. Aided Geom. Des. 4, 257-268 (1987)

15. Dyn, N., Levin, D., Micchelli, C.A.: Using parameters to increase smoothness of curves and surfaces generated by subdivision. Comput. Aided Geom. Des. 7, 129-140 (1990)

16. Gregory, J.A.: An introduction to bivariate uniform subdivision. TR/12/91, Department of Mathematics and Statistics, Brunel University, Uxbridge, Middlesex, UB8 3PH, Britain (1991)

17. Dyn, N., Levin, D.: Subdivision schemes in geometric modelling. Acta Numer. 11, $73-144$ (2002). https://doi.org/10.1017/S0962492902000028

18. Dyn, N., Levin, D.: Analysis of asymptotically equivalent binary subdivision schemes. J. Math. Anal. Appl. 193, 594-621 (1995)

19. Daubechies, I., Guskov, I., Sweldens, W.: Regularity of irregular subdivision. Constr. Approx. 15, 381-426 (1999)

20. Guglielmi, N., Manni, C., Vitale, D.: Convergence analysis of $C^{2}$ Hermite interpolatory subdivision schemes by explicit joint spectral radius. Linear Algebra Appl. 434, 884-902 (2011)

21. Dyn, N., Kuijt, F., Levin, D., Damme, R.V.: Convexity preservation of the four point interpolatory subdivision scheme. Comput. Aided Geom. Des. 16, 789-792 (1999)

22. Siddiqi, S.S., Ahmad, N.: A C ${ }^{6}$ approximating subdivision scheme. Appl. Math. Lett. 21, $722-728$ (2007) 
23. Siddiqi, S.S., Rehan, K.: Improved binary four point subdivision scheme and new corner cutting scheme. Comput. Math. Appl. 59, 2647-2657 (2010)

24. Siddiqi, S.S., Ahmad, N.: A new three-point approximating $C^{2}$ subdivision scheme. Appl. Math. Lett. 20, 707-711 (2007)

Submit your manuscript to a SpringerOpen ${ }^{\odot}$ journal and benefit from:

- Convenient online submission

$\checkmark$ Rigorous peer review

Open access: articles freely available online

- High visibility within the field

- Retaining the copyright to your article

Submit your next manuscript at $\gg$ springeropen.com 